\title{
CENTRAL CHARGES, SYMPLECTIC FORMS, AND HYPERGEOMETRIC SERIES IN LOCAL MIRROR SYMMETRY
}

\author{
SHINOBU HOSONO
}

\begin{abstract}
We study a cohomology-valued hypergeometric series which naturally arises in the description of (local) mirror symmetry. We identify it as a central charge formula for BPS states and study its monodromy property from the viewpoint of Kontsevich's homological mirror symmetry. In case of local mirror symmetry, we will identify a symplectic form, and will conjecture an integral and symplectic monodromy property of a relevant hypergeometric series of Gel'fand-Kapranov-Zelevinski type.
\end{abstract}

$\S 1$. Introduction

\section{Table of Contents}

$\S 2$. Central charge formula in terms of $w\left(x ; \frac{J}{2 \pi i}\right)$

$\S 3$. Local mirror symmetry I $-X=\widehat{\mathbf{C}^{2} / \mathbf{Z}_{\mu+1}}$

(3-1) Mirror symmetry and hyperkähler rotation

(3-2) GKZ hypergeometric series

(3-3) Example $(\mu=2)$

$\S 4$. K. Saito's differential equations

(4-1) K. Saito's system for a primitive form $\mathcal{U}_{0}(a)$

(4-2) GKZ system for a primitive form $\mathcal{U}(a)$

$\S 5$. Central charge formula and $G$-Hilb

$\S 6$. Local mirror symmetry II $-X=\widehat{\mathbf{C}^{3} / G}$

(6-1) Period integrals and GKZ systems

(6-2) Central charge formula and symplectic forms

(6-3) Prepotential and a symplectic D-brane basis

(6-4) Examples

$\S 7$. Conclusion and discussions

Appendix A. Integrating over vanishing cycles

Appendix B. Differential equation (A.3)

\section{Introduction}

Let us consider a (famous) hypergeometric series of one variable $[1, x] \in \mathbf{P}^{1}$;

$$
w(x)=\sum_{n \geq 0} \frac{(5 n) !}{(n !)^{5}} x^{n}
$$


This is a hypergeometric series of type ${ }_{4} F_{3}\left(\frac{1}{5}, \frac{2}{5}, \frac{3}{5}, \frac{4}{5} ; 1,1,1 ; x\right)$ which arises in the mirror symmetry of quintic hypersurface $X_{5} \subset \mathbf{P}^{4}$. This hypergeometric series (1.1) represents one of the period integrals of the mirror quintic $X_{5}^{\vee}$ and satisfies the following differential equation (Picard-Fuchs) equation:

$$
\left\{\theta_{x}^{4}-5^{5} x\left(\theta_{x}+\frac{4}{5}\right)\left(\theta_{x}+\frac{3}{5}\right)\left(\theta_{x}+\frac{2}{5}\right)\left(\theta_{x}+\frac{1}{5}\right)\right\} w(x)=0,
$$

where $\theta_{x}:=x \frac{d}{d x}$. See the original work by Candelas et al [CdOGP] for the description of the mirror family and its period integrals.

As it is clear in the form of differential equation, the regular singularity at $x=0$ has a distinguished property, i.e., the monodromy around this point is maximally unipotent Mor. In physics, the point $x=0$ is called a large complex structure limit and plays important roles, e.g., near this point, we evaluate the quantum corrections to the classical geometry of the $\sigma$-model on the quintic $X_{5}$. Here we focus on the construction of local solutions about $x=0$ by the classical Frobenius method;

$$
\begin{gathered}
w_{0}(x):=w(x), w_{1}(x):=\left.\frac{\partial}{\partial \rho} w(x ; \rho)\right|_{\rho=0}, \\
w_{2}(x):=\left.\frac{\partial^{2}}{\partial \rho^{2}} w(x ; \rho)\right|_{\rho=0}, w_{3}(x):=\left.\frac{\partial^{3}}{\partial \rho^{3}} w(x ; \rho)\right|_{\rho=0},
\end{gathered}
$$

where $w(x ; \rho):=\sum_{n \geq 0} \frac{\Gamma(1+5(n+\rho))}{\Gamma(1+(n+\rho))^{5}} x^{n+\rho}$. With the mirror symmetry of $X_{5}$ in $\mathbf{P}^{4}$ and $X_{5}^{\vee}$ in mind, we introduce the following cohomology-valued hypergeometric series;

$$
w\left(x ; \frac{J}{2 \pi i}\right):=w(x)+w_{1}(x)\left(\frac{J}{2 \pi i}\right)+w_{2}(x)\left(\frac{J}{2 \pi i}\right)^{2}+w_{3}(x)\left(\frac{J}{2 \pi i}\right)^{3},
$$

where $J$ is the ample, integral generator of $\operatorname{Pic}\left(X_{5}\right)=H^{1,1}\left(X_{5}\right) \cap H^{2}\left(X_{5}, \mathbf{Z}\right)$. In this form, we note that the classical Frobenius method is concisely summarized as the Taylor expansion $\left.w(x ; \rho)\right|_{\frac{J}{2 \pi i}}$ with respect to the nilpotent element $J$. Although this seems just an advantage in bookkeeping, the following observation reported in Hos indicates that we have more than that in (1.3):

Observation: Arrange the Taylor series expansion of the cohomology-valued hypergeometric series $w\left(x ; \frac{J}{2 \pi i}\right)$ as

$$
w\left(x ; \frac{J}{2 \pi i}\right)=w^{(0)}(x)+w^{(1)}(x)\left(J-\frac{c_{2}\left(X_{5}\right) J}{12}-\frac{11}{2} \frac{J^{2}}{5}\right)+w^{(2)}(x) \frac{J^{2}}{5}+w^{(3)}(x)\left(-\frac{J^{3}}{5}\right) .
$$

Then the monodromy matrices of the coefficient hypergeometric series $w^{(0)}(x)$, $w^{(1)}(x), w^{(2)}(x), w^{(3)}(x)$ are integral and symplectic (with respect to the symplectic form of the mirror, see below).

The integral and symplectic properties of the solutions $w^{(k)}(x)(k=0,1,2,3)$ stem from those of the middle homology group of the mirror $H_{3}\left(X_{5}^{\vee}, \mathbf{Z}\right)$. The point here is that we can recover these integral, symplectic properties from the series $w\left(x ; \frac{J}{2 \pi i}\right)$ through suitably arranging a basis of $H^{\text {even }}(X, \mathbf{Q})$ near the large complex structure limit. Since there is a symplectic, integral structure on $H^{\text {even }}(X, \mathbf{Q})$ which comes from $K(X)$, the Grothendieck group of algebraic vector bundles on $X$, it is natural to conjecture (Conjecture 2.2) that the cohomology-valued hypergeometric series describes a "pairing" between $K(X)$ and $H_{3}\left(X_{5}^{\vee}, \mathbf{Z}\right)$, as proposed in $\underline{\text { Hos }}$. 
The aims (and main results) of this paper are, 1) to make an interpretation of the cohomology-valued hypergeometric series from the viewpoint of homological mirror symmetry, and to give a general definition of the so-called central charge of BPS states (Conjecture 2.2, Conjecture 6.3), 2) to present supporting evidences for the interpretation in the cases from local mirror symmetry in dimensions two and three (Section 5, Section 6).

The idea of the local mirror symmetry is simply to focus on the Calabi-Yau geometry near rigid curves or (Fano) surfaces in a Calabi-Yau manifold [CKYZ]. Recently this idea has been providing us useful testing grounds for several duality symmetries proposed in string theory. Especially, when the relevant Calabi-Yau geometry is toric, remarkable progresses have been made (, see AKMV] and references therein). In this paper, for the local Calabi-Yau geometries, we will consider the crepant resolutions $\widehat{\mathbf{C}^{2} / \mathbf{Z}_{\mu+1}}$ of the two dimensional canonical singularity and also $\widehat{\mathbf{C}^{3} / G}$ with finite abelian group $G \subset S L(3, \mathbf{C})$. Although we restrict our attentions to these cases, our arguments should extend to more general local Calabi-Yau geometries.

When studying relevant Gel'fand-Kapranov-Zelevinski(GKZ) hypergeometric series, we will find its connection to the primitive forms introduced by K.Saito in the deformation theory of singularities (Proposition 4.2, Proposition 4.4). This seems to be interesting in its own right, since GKZ hypergeometric series may provide a simple way to express the 'period integrals' of the primitive forms (or oscillating integrals) in the theory of singularities.

The construction of this paper is as follows: In section 2, we give a definition of the central charge assuming Kontsevich's homological mirror symmetry. Following the observations made in $[\mathrm{Hos}$, we interpret the cohomology-valued hypergeometric series as the central charge for the cases of $X$ compact. In section 3, we consider the local mirror symmetry of $X=\mathbf{C}^{2 / \mathbf{Z}_{\mu+1}}$ and study in detail the GKZ hypergeometric series in this case. In section 4, we connect the GKZ system to K.Saito's system of differential equations which describes the deformation of singularities. In section 5, we extract a (homological) mirror map mir : $K^{c}(X) \stackrel{\sim}{\rightarrow} H_{2}(Y, \mathbf{Z})$ from our interpretation of the cohomology-valued hypergeometric series, and find a consistency with the mirror symmetry in terms of the hyperkähler rotation. In section 6 , we formulate our conjecture on cohomology-valued hypergeometric series for three dimensional cases $X=\widehat{\mathbf{C}^{3} / G}$. We also find a closed formula for the prepotential. Two explicit examples are presented to support our conjecture. In Appendix A and $\mathrm{B}$, we evaluate period integrals over vanishing cycles for the mirror geometry of $X=\widehat{\mathbf{C}^{3} / \mathbf{Z}_{3}}$. There, we will utilize a non-trivial relation between the period integral of the mirror symmetry and that of a primitive form.

Acknowledgements: The author would like to thank T. Terasoma for discussions on the GKZ hypergeometric system. He also would like to thank K. Oguiso and S.-T.Yau for valuable comments on this work. 


\section{Central charge formula in terms of $w\left(x ; \frac{J}{2 \pi i}\right)$}

Here, we will interpret the cohomology-valued hypergeometric series from the viewpoint of Kontsevich's homological mirror symmetry [Ko.

Let $X$ be a Calabi-Yau 3 fold and $Y$ be a mirror of $X$. Following Kontsevich, we consider the bounded derived category $D^{b}(C o h(X))$ of coherent sheaves (D-branes of B type) on $X$. On the other hand, for the mirror side, we consider the derived Fukaya category $\operatorname{DFuk}(Y, \beta)$ with the Kähler form viewed as a symplectic form $\beta$. The objects of the latter category consist of (graded) Lagrangian submanifolds with a flat $U(1)$ bundle on each of them (D-brane of type A) and morphisms are given by the Floer homology for Lagrangian submanifolds, and this category makes up a triangulated category (, see [FO3] for more precise definition). Kontsevich proposed that these two different categories are equivalent (as triangulated categories) when $X$ and $Y$ are mirror symmetric, and also this should be a mathematical definition of mirror symmetry. This conjecture itself is of great interest, however let us consider this conjecture at more tractable level, i.e. at the level of cohomology or K-group as shown in the second line below;

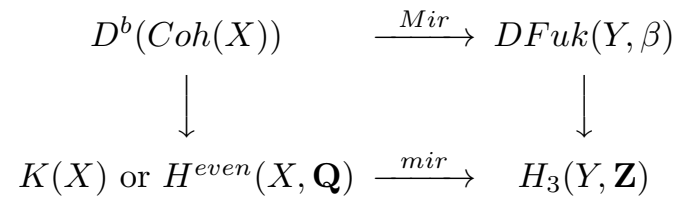

where the left vertical arrow represents the natural map from $D^{b}(C o h(X))$ to the $\mathrm{K}$ group of algebraic vector bundles $K(X)$, and its composition with the Chern character homomorphism $\operatorname{ch}(\cdot)$ if we further map to $H^{\text {even }}(X, \mathbf{Q})=\oplus_{p=0}^{3} H^{2 p}(X, \mathbf{Q})$. The right vertical arrow is given simply by taking the homology classes of the graded Lagrangian cycles. In the second line, the equivalence, Mir, of the two categories becomes simply an isomorphism, mir, between the K-group and $H_{3}(Y, \mathbf{Z})$. We should note that this is not simply an isomorphism but isomorphism with the symplectic structures, i.e.

$$
\operatorname{mir}:(K(X), \chi(E, F)) \stackrel{\sim}{\rightarrow}\left(H_{3}(Y, \mathbf{Z}), \#\left(L_{E} \cap L_{F}\right)\right),
$$

where $\chi(E, F)=\int_{X} \operatorname{ch}\left(E^{\vee}\right) \operatorname{ch}(F) \operatorname{Todd}_{X}$ and $\#\left(L_{E} \cap L_{F}\right):=\int_{Y} \mu_{L_{E}} \cup \mu_{L_{F}}$ with the Poincaré duals $\mu_{L_{E}}, \mu_{L_{F}} \in H^{3}(Y, \mathbf{Z})$ of the mirror homology cycles $L_{E}:=$ $\operatorname{mir}(E), L_{F}:=\operatorname{mir}(F)$. Here we remark that the Euler number $\chi(E, F)$ is antisymmetric due to Serre duality and $K_{X}=0$, and also non-degenerate. Thus $\chi(E, F)$ introduces a symplectic structure on $K(X)$, which is the mirror of the symplectic structure on $H_{3}(Y, \mathbf{Z})$.

In the diagram (2.1), we assume that a complex structure is fixed to define $D^{b}(\operatorname{Coh}(X))$. Correspondingly the symplectic form (Kähler form) $\beta$, determined by the mirror map, is fixed in the right hand side. On the other hand, we may change the (complexified) Kähler class of $X$ which corresponds to the complex structure moduli of $Y$ under mirror map. Changing the (complexified) Kähler structure amounts to changing the polarization and thus results in varying the stability condition on the sheaves on $X$. This change of the stability (ח-stability) condition has been studied in Do as a stability of BPS D-branes and its mathematical aspects are elaborated in $\mathrm{Br}$. Here, we will not go into the detailed definition of the П-stability, but we will propose a closed formula for the central charge which is indispensable for an explicit description of the $\Pi$ stability. 
Definition 2.1. (Central charge formula.) Assume $K(X)$ is torsion free, and let $E_{1}, \cdots, E_{r}$ be a $\mathbf{Z}$ basis of $K(X)$. Let $\Omega\left(Y_{x}\right)$ be a holomorphic 3 -form of the mirror family $\left\{Y_{x}\right\}_{x \in \mathcal{B}}$ of $X$. Under the mirror symmetry (2.1), we define the following $\mathcal{Z}_{x}$ as an element in $K(X) \otimes \mathbf{C}\{x\}[\log x]$,

$$
\mathcal{Z}_{x}:=\sum_{i, j} \int_{\operatorname{mir}\left(E_{i}\right)} \Omega\left(Y_{x}\right) \chi^{i j} E_{j}^{\vee}
$$

with $\left(\chi^{i j}\right):=\left(\chi\left(E_{i}, E_{j}\right)\right)^{-1}$. Then the central charge of $F \in K(X)$ is defined by

$$
Z_{t}(F)=\int_{X} \operatorname{ch}(F) \operatorname{ch}\left(\mathcal{Z}_{x}\right) \operatorname{Todd}_{X}
$$

where $t=t(x)$ is the mirror map representing the (complexified) Kähler moduli of $X$.

In the above definition, it should be noted that $\mathcal{Z}_{x}$ does not depend on the choice of a basis $E_{1}, \cdots, E_{r}$. Also the central charge $Z_{t}(F)$ contains full 'quantum corrections' ( cf. the asymptotic formula given in [Do ).

We will connect our hypergeometric series $w\left(x ; \frac{J}{2 \pi i}\right)$ to the central charge above. Before doing this, we remark that, in the mirror symmetry of Calabi-Yau hypersurfaces due to Batyrev Ba1, the hypergeometric series (1.1) is naturally generalized to Gel'fand-Kapranov-Zelevinski(GKZ) hypergeometric series of multi variables $x_{1}, \cdots, x_{r}$ GKZ1 Ba2. Using the GKZ hypergeometric series, and also suitable integral, (semi-)ample generators $J_{1}, \cdots, J_{r}$ of $H^{1,1}(X) \cap H^{2}(X, \mathbf{Z})$, we have a cohomology-valued hypergeometric series $w\left(x ; \frac{J}{2 \pi i}\right)$ as a generalization of (1.3), see Sect.2 of [Hos for more details.

Conjecture 2.2. The cohomology-valued hypergeometric series 1.3) gives the central charge $\operatorname{ch}\left(\mathcal{Z}_{x}\right)$;

$$
w\left(x_{1}, \cdots, x_{r} ; \frac{J_{1}}{2 \pi i}, \cdots, \frac{J_{r}}{2 \pi i}\right)=\sum_{i, j} \int_{\operatorname{mir}\left(E_{i}\right)} \Omega\left(Y_{x}\right) \chi^{i j} \operatorname{ch}\left(E_{j}^{\vee}\right) .
$$

Using this, and also the mirror map $t=t(x)$, we can write the central charge $Z_{t}(F)$ of $F \in K(X)$ as

$$
Z_{t}(F)=\int_{X} \operatorname{ch}(F) w\left(x ; \frac{J}{2 \pi i}\right) \operatorname{Todd}_{X} .
$$

Here we note that the hypergeometric series has a finite radius of convergence and shows a monodromy property when it is analytically continued around its (regular) singularities. As noticed by Kontsevich, this monodromy property should be mirrored to some linear (symplectic) transformations on $\operatorname{ch}\left(E_{i}\right)$ which come from Fourier-Mukai transforms on $D^{b}(\operatorname{Coh}(X))$. If we postulate that the cohomologyvalued hypergeometric series has an invariant meaning under these monodromy actions, our cohomology-valued hypergeometric series $w\left(x ; \frac{J}{2 \pi i}\right)$ provides a connection between these two different 'monodromy' transforms in both sides. The conjectural formula (2.4) has been tested in case $X$ is an elliptic curve, (lattice polarized) K3 surfaces, and several Calabi-Yau hypersurfaces in $\mathrm{Hos}$. Cohomology-valued hypergeometric series are utilized also in [Gi][LLY] Sti] in a slightly different form. We remark that, for our Conjecture 2.2 to work, the definition given in $\mathrm{Hos}$ is crucial. 
As studied in $[\mathrm{Mu}$. for the cases of $\mathrm{K} 3$ surfaces and abelian varieties, and in $\mathrm{Or}$ for general, the Fourier-Mukai transform is a self-equivalence of the category $D^{b}(\operatorname{Coh}(X))$ which takes the form

$$
\Phi^{\mathcal{P}}(\cdot)=\mathbf{R} p_{2 *}\left(p_{1}^{*}(\cdot) \stackrel{\mathrm{L}}{\otimes} \mathcal{P}\right)
$$

where $\mathcal{P}$ is an object in $D^{b}(\operatorname{Coh}(X \times X))$, called the kernel, and $p_{1}$ and $p_{2}$ are, respectively, the natural projections to the first and the second factor from $X \times$ $X$. Due to a result in $\mathrm{Or}$, we may always assume the above form, i.e., there exists a suitable kernel $\mathcal{P}$, for any equivalence $\Phi: D^{b}(\operatorname{Coh}(X)) \simeq D^{b}(\operatorname{Coh}(X))$ as triangulated category. It is rather easy to see that the monodromy transforms around the large complex structure limit are given by tensoring invertible sheaves, which may be expressed by the kernels;

$$
\mathcal{P}: \cdots \rightarrow 0 \rightarrow \mathcal{O}_{\Delta} \times p_{2}^{*}\left(\mathcal{O}_{X}(D)\right) \rightarrow 0 \cdots,
$$

with $D \in \operatorname{Pic}(X)$ and $\Delta$ representing the diagonal in $X \times X$. Kontsevich predicted that a monodromy transform associated to a vanishing cycle, Picard-Lefschetz transform, has its mirror FM transform with its kernel,

$$
\mathcal{P}: \cdots \rightarrow 0 \rightarrow \mathcal{O}_{X \times X} \rightarrow \mathcal{O}_{X} \rightarrow 0 \cdots .
$$

Seidel and Thomas $[\mathrm{ST}$ (and Horja $[$ Hor ) generalized the above kernel associating it to so-called spherical objects $\mathcal{E} \in D^{b}(\operatorname{Coh}(X))$ with the defining property: $\operatorname{Ext}^{i}(\mathcal{E}, \mathcal{E})=0(i \neq 0, n), \mathbf{C}(i=0, n)$ where $n=\operatorname{dim} X$. For each spherical object, we have a kernel given by the mapping cone;

$$
\mathcal{P}=\operatorname{Cone}\left(\mathcal{E}^{\vee} \otimes^{\mathbf{L}} \mathcal{E} \rightarrow \mathcal{O}_{\Delta}\right) .
$$

The equivalence $\Phi^{\mathcal{P}}$ is called Seidel-Thomas twist. We will see these equivalences in the corresponding monodromy property of our hypergeometric series.

\section{Local mirror symmetry $\mathbf{I}-X=\widehat{\mathbf{C}^{2} / \mathbf{Z}_{\mu+1}}$}

In this section and the subsequent sections, we will test our Conjecture 2.2 for the case of mirror symmetry of non-compact toric Calabi-Yau manifolds (local mirror symmetry). Batyrev's idea of mirror symmetry Ba1] still makes sense for such non-compact toric Calabi-Yau manifolds as well as the attractive proposal by Strominger-Yau-Zaslow(SYZ) [SYZ, which is closely related to the homological mirror symmetry (2.1). Mirror symmetry of non-compact toric Calabi-Yau manifolds and also Fano varieties are formulated in a language of Landau-Ginzburg theory in [HIV].

(3-1) Mirror symmetry and hyperkähler rotation. Let us consider the minimal resolution of a two dimensional simple singularity; $X=\widehat{\mathbf{C}^{2} / \mathbf{Z}_{\mu+1}}$. This is an example of two dimensional, non-compact, toric Calabi-Yau manifold. Two dimensional Calabi-Yau manifolds are hyperkähler, and it is known that the mirror symmetry of them is well-understood by the hyperkähler rotation, see e.g. GW] Huy. Our minimal resolution $X$ has a natural hyperkähler structure, and therefore its mirror is $X$ itself with a different complex structure after a suitable rotation. To describe the mirror symmetry, let us first write the quotient $\mathbf{C} / \mathbf{Z}_{\mu+1}$ by a hypersurface $U V=W^{\mu+1}$ in $\mathbf{C}^{3}$. Bowing up the singularities at the origin 
$\mu$ times results in the minimal resolution $X$, and thereby we introduce exceptional curves $C_{i} \cong \mathbf{P}^{1}(i=1, \cdots, \mu)$. On the other hand, we may deform the defining equation $U V=W^{\mu+1}$ to $U V=a_{1}+a_{2} W+\cdots+a_{\mu+2} W^{\mu+1}$ with introducing finite sizes to vanishing cycles $L_{i} \approx S^{2}(\mu=1, \cdots, \mu)$. Note that the number of vanishing cycles are given by the Milnor number $\mu=\operatorname{dim} R_{J}$, where $R_{J}$ is the Jacobian ring of the singularity $U V=W^{\mu+1}$. The vanishing cycles are Lagrangians, and become holomorphic cycles under a suitable hyperkähler rotation. The holomorphic geometry after the rotation is bi-holomorphic to the blown-up geometry of $X$. If we forget about the role of the $B$-fields, this describes the mirror symmetry of $X$. (See e.g. Huy and references therein for full details of the mirror symmetry via the hyperkähler rotation.) Here we note that the intersection form of these vanishing cycles are given in both holomorphic and Lagrangian geometry by

$$
\left(C_{i} \cdot C_{j}\right)=\left(\# L_{i} \cap L_{j}\right)=-\mathcal{C}_{i j},
$$

where $\mathcal{C}_{i j}(1 \leq i, j \leq \mu)$ is the Cartan matrix for the root system of $A_{\mu}$.

(3-2) GKZ hypergeometric series. The minimal resolution $X=\widehat{\mathbf{C}^{2 / \mathbf{Z}_{\mu+1}}}$ is a (non-compact) toric variety whose resolution is described by a two dimensional fan $\Sigma$ with its integral generators for one dimensional cones (see Fig.1);

$$
A=\left\{\nu_{1}=(1,0), \nu_{2}=(1,1), \cdots, \nu_{\mu+2}=(1, \mu+1)\right\} .
$$

The half-lines $\overline{o \nu}_{i}(i=1, \cdots, \mu+2)$ from the origin $o=(0,0)$ constitute the one dimensional cones of the resolution diagram $\Sigma$. In Batyrev's mirror symmetry, the resolution diagram of $X$, up to flop operations, is identified with the Newton polytope of the defining equation of its mirror $Y$. This construction has been extended to the cases of non-compact toric Calabi-Yau maniflods in [CKYZ HIV], and for $X=\widehat{\mathbf{C}^{2} / \mathbf{Z}_{\mu+1}}$, the mirror $Y$ is given by $U^{2}+V^{2}+f_{\Sigma}(W)=0 \subset \mathbf{C}^{2} \times \mathbf{C}^{*}$ with

$$
f_{\Sigma}(W)=a_{1}+a_{2} W^{1}+a_{3} W^{2}+\cdots+a_{\mu+2} W^{\mu+1}
$$

where $(U, V, W) \in \mathbf{C}^{2} \times \mathbf{C}^{*}$. In the case of local mirror symmetry, the meaning of the period integrals of holomorphic two form becomes less clear than the compact cases. Here we consider the following integral for a cycle $\gamma \in H_{3}\left(\mathbf{C}^{2} \times \mathbf{C}^{*} \backslash\left(U^{2}+\right.\right.$ $\left.\left.V^{2}+f_{\Sigma}(a ; W)=0\right), \mathbf{Z}\right)$ :

$$
\Pi_{\gamma}(a):=\int_{\gamma} \frac{1}{U^{2}+V^{2}+f_{\Sigma}(a ; W)} d U d V \frac{d W}{W} .
$$

In the next section, we will relate this 'period integral' to K. Saito's primitive form (or Gel'fand-Leray form) in the deformation theory of singularities Sa AGV]. Here we set up a hypergeometric differential equations (GKZ system) satisfied by $\Pi_{\gamma}(a)$. This GKZ hypergeometric system is also referred to as $A$-hypergeometric system since it is described by the set $A$ through the lattice of relations,

$$
L=\left\{\left(l_{1}, l_{2}, \cdots, l_{\mu+2}\right) \mid l_{1} \nu_{1}+l_{2} \nu_{2}+\cdots+l_{\mu+2} \nu_{\mu+2}=(0,0)\right\} .
$$

Using this lattice of relations, the system is written as

$$
\square_{l} \Pi_{\gamma}(a)=0(l \in L), \quad \mathcal{Z}_{i} \Pi_{\gamma}(a)=0(i=1,2),
$$

where

$$
\square_{l}=\left(\frac{\partial}{\partial a}\right)^{l^{+}}-\left(\frac{\partial}{\partial a}\right)^{l^{-}},\left(\begin{array}{c}
\mathcal{Z}_{1} \\
\mathcal{Z}_{2}
\end{array}\right)=\left(\begin{array}{c}
\theta_{1}+\theta_{2}+\cdots+\theta_{\mu+2} \\
\theta_{2}+2 \theta_{3} \cdots+(\mu+1) \theta_{\mu+2}
\end{array}\right)
$$


with $l=l^{+}-l^{-}\left(l_{i}^{ \pm}:=\frac{1}{2}\left(l_{i} \pm\left|l_{i}\right|\right)\right.$ and $\theta_{k}=a_{k} \frac{\partial}{\partial a_{k}}$. From the formal solutions of this system in [GKZ1, it is easy to write down our $w\left(x ; \frac{J}{2 \pi i}\right)$ (, see (3-3) below). An important aspect of this system is that there is a natural toric compactification $\mathcal{M}_{S e c(\Sigma)}$ of the parameter space $\left\{\left(a_{1}, \cdots, a_{\mu+2}\right) \in\left(\mathbf{C}^{*}\right)^{\mu+2} /\left(\mathbf{C}^{*}\right)^{2}\right\}$ in terms of the secondary fan $\operatorname{Sec}(\Sigma)$ (, see GKZ2 and references therein), where the quotient by $\left(\mathbf{C}^{*}\right)^{2}$ is represented by the (scaling) operators $\mathcal{Z}_{1}$ and $\mathcal{Z}_{2}$. This compactification plays an important role in the applications of mirror symmetry to Gromov-Witten invariants, since the large radius limit appears as an intersection point of the boundary toric divisors. Connecting the GKZ system to K. Saito's differential equations in singularity theory, we will see in section 4 that this compactification will also provide a natural way to compactify the deformation space of singularities which is local in nature (, see (4-1) for a brief description of the deformation space).
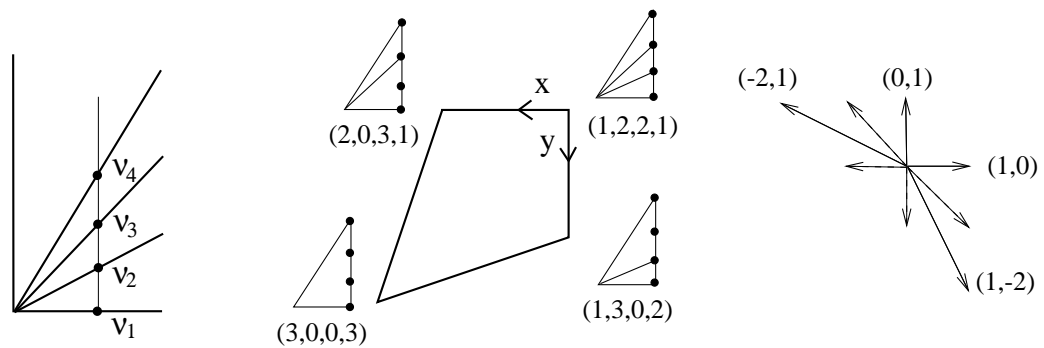

Fig.1. The resolution diagram (left), the secondary polytope (middle), and the secondary fan $\operatorname{Sec}(\Sigma)$ (right) for $\mu=2$. The secondary polytope has its vertices parametrized by (regular) triangulations of the polytope as shown. For a triangulation $T$, the corresponding vertex is determined by a vector $v_{T}=\left(\varphi_{T}\left(\nu_{1}\right), \varphi_{T}\left(\nu_{2}\right), \varphi_{T}\left(\nu_{3}\right), \varphi_{T}\left(\nu_{4}\right)\right)$ with $\varphi_{T}\left(\nu_{i}\right)=\sum_{\nu_{i} \prec \sigma} \operatorname{Vol}(\sigma)$. As we see in this example $(\mu=2)$, the convex hull of these vertices lies on $L_{\mathbf{R}}+v_{T}$ with a vector $v_{T}$, where $L_{\mathbf{R}}=L \otimes \mathbf{R}$. Normal cones of the secondary polytope determines the secondary fan.

(3-3) Example $(\mu=2)$. Here we present some explicit calculations of cohomologyvalued hypergeometric series for the case $\mu=2$, instead of giving general formulas valid for any $\mu$. In this case, the cohomology-valued hypergeometric series is simple and takes the following form;

$$
w\left(x ; y ; \frac{J_{1}}{2 \pi i}, \frac{J_{2}}{2 \pi i}\right)=\left.w\left(x ; y ; \rho_{1}, \rho_{2}\right)\right|_{\rho_{1}=\frac{J_{1}}{2 \pi i}, \rho_{2}=\frac{J_{2}}{2 \pi i}},
$$

where $w\left(x ; y ; \rho_{1}, \rho_{2}\right)=\sum_{n, m \geq 0} c\left(n+\rho_{1}, m+\rho_{2}\right) x^{n+\rho_{1}} y^{m+\rho_{2}}$ with

$$
c(n, m)=1 /(\Gamma(1+n) \Gamma(1-2 n+m) \Gamma(1+n-2 m) \Gamma(1+m)) .
$$

$J_{1}$ and $J_{2}$ are semi-ample classes which are dual to the exceptional curves $C_{1}$ and $C_{2}$ (, i.e. the toric divisors $D_{\nu_{2}}$ and $D_{\nu_{3}}$ ), respectively. The local parameters $x:=\frac{a_{1} a_{3}}{a_{2}^{2}}, y:=\frac{a_{2} a_{4}}{a_{3}^{2}}$ are depicted in Fig.1. Here we remark that the secondary polytope in Fig.1 sits on a translation of $L_{\mathbf{R}}$, i.e. $L_{\mathbf{R}}+v_{T}$, and the summation in $\sum_{n, m>0} c(m, m) x^{m} y^{n}$ is in fact that over the integral points inside the dual of the normal cone from the vertex $v_{T}=(1,2,2,1)$. We may recognize this fact in the relation $x^{n} y^{m}=a^{n l^{(1)}+m l^{(2)}}$, where by definition $l^{(1)}=(1,-2,1,0)$ and $l^{(2)}=$ 
$(0,1,-2,1)$ generate the dual of the normal cone form the vertex $v_{T}$. We remark also that the coefficient $c(n, m)$ are determined from the entries of $n l^{(1)}+m l^{(2)}$;

$$
(n m)\left(\begin{array}{cccc}
1 & -2 & 1 & 0 \\
0 & 1 & -2 & 1
\end{array}\right)=\left(\begin{array}{cccc}
n & -2 n & n & 0 \\
0 & m & -2 m & m
\end{array}\right),
$$

where the row vectors determine the arguments of the gamma functions in the denominator of $c(n, m)$. In this form, it is rather clear how the formula generalizes to arbitrary $\mu$ (, see [CKYZ, dOFS] for example).

Since the ring $H^{\text {even }}(X, \mathbf{Z})$ is generated by $1, J_{1}, J_{2}$, we have the expansion;

$$
w\left(\vec{x}, \frac{\vec{J}}{2 \pi i}\right)=1+w_{1}(x, y) J_{1}+w_{2}(x, y) J_{2}
$$

with $w_{1}(x, y)=\frac{1}{2 \pi i} \log x+\cdots, w_{2}(x, y)=\frac{1}{2 \pi i} \log y+\cdots$. The mirror map is defined from the relations,

$$
q_{1}:=e^{2 \pi i w_{1}(x, y)}=x\left(1+g_{1}(x, y)\right), q_{2}:=e^{2 \pi i w_{2}(x, y)}=y\left(1+g_{2}(x, y)\right),
$$

where $g_{1}(x, y), g_{2}(x, y)$ represent powerseries of $x$ and $y$. Then $t_{1}:=\frac{1}{2 \pi i} \log q_{1}(=$ $\left.w_{1}(x, y)\right)$ and $t_{2}:=\frac{1}{2 \pi i} \log q_{2}\left(=w_{2}(x, y)\right)$ are the complexified Kähler moduli and measure the volumes of the exceptional curves $C_{1}$, and $C_{2}$, respectively. The inverse relation $x=x\left(q_{1}, q_{2}\right), y=y\left(q_{1}, q_{2}\right)$ of (3.4) is often referred to as the mirror map, and has the following properties (see Proposition 4.4 for a proof);

\section{Proposition 3.1.}

1) The mirror map $x=x\left(q_{1}, q_{2}\right), y=y\left(q_{1}, q_{2}\right)$ is rational and is expressed by $x=$ $\frac{a_{1} a_{3}}{a_{2}^{2}}, y=\frac{a_{2} a_{4}}{a_{3}^{2}}$ with $a_{i}$ 's determined through

$$
a_{1}+a_{2} W+a_{3} W^{2}+a_{4} W^{3}=(1+W)\left(1+q_{1} W\right)\left(1+q_{1} q_{2} W\right) .
$$

Concretely, it has the form;

$$
x=\frac{q_{1}\left(1+q_{2}+q_{1} q_{2}\right)}{\left(1+q_{1}+q_{1} q_{2}\right)^{2}}, y=\frac{q_{2}\left(1+q_{1}+q_{1} q_{2}\right)}{\left(1+q_{1}+q_{1} q_{2}\right)^{2}} .
$$

2) The discriminant of the GKZ system (3.3) consists of three components; $x=$ $0, y=0$ and $\operatorname{dis}(x, y)=0$ with

$$
\operatorname{dis}(x, y)=1-4 x-4 y+18 x y-27 x^{2} y^{2}=\frac{\left(1-q_{1}\right)^{2}\left(1-q_{2}\right)^{2}\left(1-q_{1} q_{2}\right)^{2}}{\left(1+q_{1}+q_{1} q_{2}\right)^{2}\left(1+q_{2}+q_{1} q_{2}\right)^{2}} .
$$

It is easy to see that $x=0, y=0$ are the toric boundary divisors whose intersection point define the large complex structure. Over the zeros of the discriminant $\operatorname{dis}(x, y)$, we see vanishing cycles in $f_{\Sigma}(a, W)+U^{2}+V^{2}=0 \subset \mathbf{C}^{2} \times \mathbf{C}^{*}$. In fact, in the holomorphic picture, $q_{1}=1\left(q_{2}=1\right)$ represents a vanishing volume limit of the exceptional curve $C_{1}\left(C_{2}\right)$. After a hyperkähler rotation, these vanishing volumes are identified as the corresponding vanishing of the Lagrangian cycles $L_{1}, L_{2}$.

We remark that the above Proposition [3.1 and also the interpretation made above generalize to arbitrary $\mu$ in a straightforward way. For example, the relation (3.5) should be read,

$$
\sum_{k=0}^{k=\mu+1} a_{k+1} W^{k}=(1+W)\left(1+q_{1} W\right)\left(1+q_{1} q_{2} W\right) \cdots\left(1+q_{1} \cdots q_{\mu} W\right),
$$

and the discriminant is essentially given by the difference products the roots $1,-1 / q_{1}$, $\cdots,-1 /\left(q_{1} \cdots q_{\mu}\right)$ (, see Proposition 4.1 and 4.4 ). 

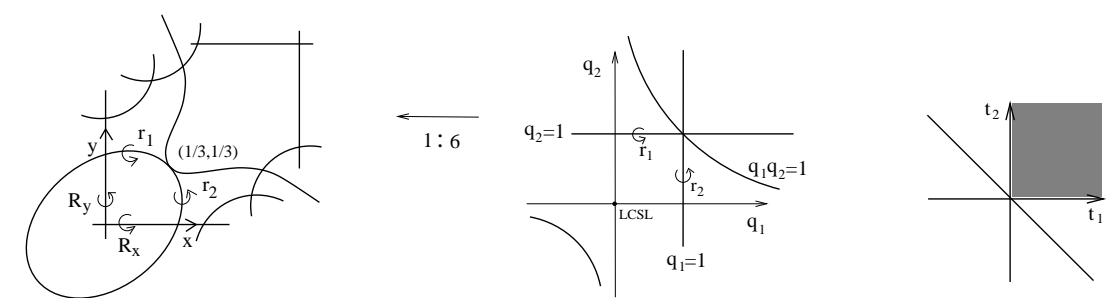

Fig.2. The discriminant $\operatorname{dis}(x, y)=0$ in $\hat{\mathcal{M}}_{\operatorname{Sec}(\Sigma)}$ (left), the mirror map (3.6) from the $q_{1} q_{2}$-plane to the $x y$-plane (middle), and the complexified Kähler moduli $t_{1}, t_{2}$ with the complexified Kähler cone (right). The discriminant is an elliptic curve with a node at $(x, y)=$ $\left(\frac{1}{3}, \frac{1}{3}\right)$, which corresponds to $\left(q_{1}, q_{2}\right)=(1,1)$. The mirror map is $6: 1$ at generic $\left(q_{1}, q_{2}\right)$. Over the discriminant, it is $3: 1$ and the inverse image of dis $(x, y)=0$ consists of the three lines $q_{1}=1, q_{2}=1, q_{1} q_{2}=1$ in the q-plane.

In the rest of this subsection, we look at the mirror map (3.6), and summarize its monodromy property. Let us first note that the moduli space $\mathcal{M}_{\operatorname{Sec}(\Sigma)}$ is a two dimensional singular toric variety, and may be desingularized to $\hat{\mathcal{M}}_{\operatorname{Sec}(\Sigma)}=$ $B l_{4}\left(\mathbf{P}^{1} \times \mathbf{P}^{1}\right)$ after blowing up four points (, see the dashed lines in Fig.1). Then the discriminant $\operatorname{dis}(x, y)=0$ describes a nodal elliptic curve written in Fig.2. Note that the mirror map form $\left(q_{1}, q_{2}\right)$ to $(x, y)$ is six to one at generic points because (3.6) is invariant under the reflections;

$$
r_{1}: q_{1} \rightarrow 1 / q_{1}, q_{2} \rightarrow q_{1} q_{2} \quad, \quad r_{2}: q_{2} \rightarrow q_{1} q_{2} \quad, \quad q_{1} \rightarrow 1 / q_{2} .
$$

which satisfy $r_{1}^{2}=r_{2}^{2}=\left(r_{1} r_{2}\right)^{3}=1$ and thus generate the symmetric group of order 3. By making an analytic continuation of the hypergeometric series, we can derive the above invariance group actions (3.7) as the monodromy actions for the loops $r_{1}$ and $r_{2}$ depicted in Fig.2. Together with the monodromy matrices $R_{x}, R_{y}$ about the large complex structure limit, we summarize the monodromy generators with respect to a basis ${ }^{t}\left(1, w_{1}(x, y), w_{2}(x, y)\right)={ }^{t}\left(1, \frac{1}{2 \pi i} \log q_{1}, \frac{1}{2 \pi i} \log q_{2}\right)$;

$$
r_{1}=\left(\begin{array}{ccc}
1 & 0 & 0 \\
0 & -1 & 0 \\
0 & 1 & 1
\end{array}\right), r_{2}=\left(\begin{array}{ccc}
1 & 0 & 0 \\
0 & 1 & 1 \\
0 & 0 & -1
\end{array}\right) ; R_{x}=\left(\begin{array}{ccc}
1 & 0 & 0 \\
1 & 1 & 0 \\
0 & 0 & 1
\end{array}\right), R_{y}=\left(\begin{array}{ccc}
1 & 0 & 0 \\
0 & 1 & 0 \\
1 & 0 & 1
\end{array}\right) \text {. }
$$

In general, we may state the monodromy property as follows;

Proposition 3.2. The mirror map (3.6) uniformizes the solutions of the GKZ system (3.3) with the symmetric group $\mathcal{S}_{\mu}$ of order $\mu$, up to the shifts $w_{i}\left(x_{1}, \cdots, x_{\mu}\right) \mapsto$ $w_{i}\left(x_{1}, \cdots, x_{\mu}\right)+1(i=1, \cdots, \mu)$. In other words, the mirror coordinate $q_{1}, \cdots, q_{\mu}$ uniformizes the hypergeometric series with the symmetric group $\mathcal{S}_{\mu}$ of order $\mu$.

The above result seems to be interesting from the viewpoint of the uniformization of hypergeometric series $\underline{Y_{0}}$, since our GKZ system provides an infinite number of examples for which multi-valued hypergeometric series are uniformizable. However the result itself is not surprising, as we will show in the next section that our GKZ system has a close relation to K. Saito's differential equations for which the monodromy property is well-studied. 


\section{K.Saito's differential equations}

In the deformation theory of a singularity, we have a notion of so-called primitive form which satisfies a set of differential equations, K. Saito's differential equations $[\underline{\mathrm{Sa}}$. Primitive form is also referred to as Gel'fand-Leray form $\mathbf{A G V}$. Here we briefly introduce the primitive form and K. Saito's differential equations, and then connect them to the 'period integral' (3.1) and the GKZ system (3.3).

(4-1) K.Saito's system for a primitive form $\mathcal{U}_{0}(a)$. Let us first note that, in the deformation of the singularity $U^{2}+V^{2}+W^{\mu+1}$, the polynomial equation $f_{\Sigma}(a, W)+U^{2}+V^{2}=0$ will be considered in $\mathbf{C}^{3}$ and the deformation parameters are set to $a_{\mu+2}=1, a_{\mu+1}=0$ by a coordinate change of $W$. Namely we take the defining equation of the form $f_{\Sigma}(a, W)+U^{2}+V^{2}=a_{1}+f_{1}(a, U, V, W)$ with

$$
f_{1}(a, U, V, W)=a_{2} W+\cdots+a_{\mu} W^{\mu-1}+W^{\mu+1}+U^{2}+V^{2},
$$

and regard the parameters $a_{1}, a_{2}, \cdots, a_{\mu}$ as giving a deformation of the singularity $W^{\mu+1}+U^{2}+V^{2}=0 \subset \mathbf{C}^{2}$ at the origin. Since the parameter $a_{1}$ plays a distinguished role from the others, we set the local parameters $\left(a_{2}, \ldots, a_{\mu}\right)$ as a coordinate of $T:=\mathbf{C}^{\mu-1}$. The full parameters $\left(a_{1}, a_{2}, \cdots, a_{\mu}\right)$ will be regarded as a coordinate of $S:=\mathbf{C} \times T$. We consider the product space $\mathfrak{X}:=\mathbf{C}^{3} \times T$ with coordinate $\left(U, V, W, a_{2}, \cdots, a_{\mu}\right)$. Then we have a natural map $\varphi: \mathfrak{X} \rightarrow S$ by

$$
\left(U, V, W, a_{2}, \cdots, a_{\mu}\right) \mapsto\left(-f_{1}(a, U, V, W), a_{2}, \cdots, a_{\mu}\right) .
$$

This map plays important roles in describing the deformation of the singularity. Consider the sheaf $\Omega_{\mathfrak{X} / T}^{p}$ of germs of relative holomorphic $p$ forms for the natural projection $\pi: \mathfrak{X}=\mathbf{C}^{3} \times T \rightarrow T$. We may consider the following sheaves on $S$,

$\mathcal{H}^{(0)}=\varphi_{*} \Omega_{\mathfrak{X} / T}^{3} / d f_{1} \wedge d\left(\varphi_{*} \Omega_{\mathfrak{X} / T}^{1}\right), \quad \mathcal{H}^{(-1)}=\varphi_{*} \Omega_{\mathfrak{X} / T}^{2} /\left(d f_{1} \wedge \varphi_{*} \Omega_{\mathfrak{X} / T}^{1}+d\left(\varphi_{*} \Omega_{\mathfrak{X} / T}^{1}\right)\right)$.

A primitive form $\zeta$ is an element in $H^{0}\left(S, \mathcal{H}^{(0)}\right)$ satisfying certain conditions (, see Sa for details). Instead of $\zeta$, hereafter, we consider its image $\mathcal{U}_{0}$ in $H^{0}\left(S, \mathcal{H}^{(-1)}\right)$ under an isomorphisms $\mathcal{H}^{(0)} \cong \mathcal{H}^{(-1)}$ (see [Sa]), which we may write explicitly as

$$
\mathcal{U}_{0}(a)=\operatorname{Res}_{\left\{a_{1}+f_{1}=0\right\}}\left(\frac{d U \wedge d V \wedge d W}{a_{1}+f_{1}(a, U, V, W)}\right) .
$$

In this from, primitive form is called also as Gel'fand-Leray form in the study of oscillating integrals (, see e.g. AGV]). We note that a similarity of $\mathcal{U}_{0}(a)$ to our 'period integral' (3.1), although, in (3.1), we do not set $a_{\mu+2}=1, a_{\mu+1}=0$ but consider torus actions $\left(\mathbf{C}^{*}\right)^{2}$ instead.

K.Saito's system is defined as a set of differential equations satisfied by the primitive form $\mathcal{U}_{0}(a)=\mathcal{U}_{0}\left(a_{1}, \cdots, a_{\mu}\right)$;

$$
\begin{aligned}
P_{i j} \mathcal{U}_{0}(a) & =\left\{\frac{\partial^{2}}{\partial a_{i} \partial a_{j}}-\nabla_{\frac{\partial}{\partial a_{i}}} \frac{\partial}{\partial a_{j}}-\left(\frac{\partial}{\partial a_{i}} * \frac{\partial}{\partial a_{j}}\right) \frac{\partial}{\partial a_{1}}\right\} \mathcal{U}_{0}(a)=0, \\
Q\left(\frac{\partial}{\partial a_{i}}\right) \mathcal{U}_{0}(a) & =\left\{w\left(\frac{\partial}{\partial a_{i}}\right) \frac{\partial}{\partial a_{1}}-N\left(\frac{\partial}{\partial a_{i}}\right)+\frac{3}{2} \frac{\partial}{\partial a_{i}}\right\} \mathcal{U}_{0}(a)=0,
\end{aligned}
$$

see $[\mathrm{Sa}$ for detailed definitions. This system is defined for a general setting in the deformation theory of singularities, and also known to define a holonomic system. A proof of the following proposition may be found in Appendix by Ambai in Oda1, 
S. HOSONO

for example. Although, we restrict our attention to $A_{\mu}$ case, similar results are known also for other ( $D$ or $E$ ) types of singularities.

Proposition 4.1. Let $\beta_{0}(a), \cdots, \beta_{\mu}(a)$ be the roots of $\psi_{0}(W):=a_{1}+a_{2} W+\cdots+$ $a_{\mu} W^{\mu-1}+W^{\mu+1}$ which satisfy $\beta_{0}(a)+\cdots+\beta_{\mu}(a)=0$. Then the space of the solutions of K.Saito's system is generated by

$$
1, \beta_{0}(a)-\beta_{1}(a), \cdots, \beta_{\mu-1}(a)-\beta_{\mu}(a) .
$$

The system has a regular singularity at the discriminant locus

$$
\operatorname{dis} \psi_{0}(a)=\Pi_{1 \leq i, j \leq \mu}\left(\beta_{i}(a)-\beta_{j}(a)\right)^{2}=0,
$$

and the monodromy group about the discriminant coincides with the symmetric group $S_{\mu}$ of order $\mu$ acting as permutations among the roots $\beta_{i}(a)$ 's.

As we have noted in (3-1), $\mu$ vanishing cycles appears in the deformation of the singularity. Now it is easy to find that the above solutions $\alpha_{i}:=\beta_{i}(a)-$ $\beta_{i-1}(a)$ represent the integrals $\int_{C} \mathcal{U}_{0}(a)$ over the corresponding vanishing cycles (, see Appendix A). Also we note that there is a residue pairing $I\left(\alpha_{i}, \alpha_{j}\right)$ among the solutions which reproduces the intersection pairing $\# L_{i} \cap L_{j}$ among the vanishing cycles $\mathrm{Sa}$.

(4-2) GKZ system for a primitive form $\mathcal{U}(a)$. As defined above, the primitive form $\mathcal{U}_{0}(a)$ is parametrized by $\left(a_{1}, \cdots, a_{\mu}\right) \in S$ by setting $a_{\mu+1}=0, a_{\mu+2}=1$ in the defining equation $f_{\Sigma}(a, W)+U^{2}+V^{2}$. Instead of this, one may consider a natural torus actions $\left(\mathbf{C}^{*}\right)^{2}$ on $\left(a_{1}, \cdots, a_{\mu+2}\right)$ by $a_{i} \mapsto \lambda a_{i}$ and $a_{i} \mapsto \lambda^{i-1} a_{i}$ $\left(\lambda \in \mathbf{C}^{*}\right)$. With this slight change of the parameters ('gauge'), we may connect K.Saito's system to a GKZ system. Let us define a period integral of the primitive form

$$
\int_{C} \mathcal{U}(a)=\int_{C} \operatorname{Res}_{f_{\Sigma}(W)+U^{2}+V^{2}=0}\left(\frac{d W \wedge d U \wedge d V}{f_{\Sigma}(a, W)+U^{2}+V^{2}}\right),
$$

where $C$ is a two cycle on $f_{\Sigma}(a, W)+U^{2}+V^{2}=0 \subset \mathbf{C}^{3}$ and $f_{\Sigma}(a, W)=a_{1}+$ $a_{2} W+\cdots+a_{\mu+2} W^{\mu+1}$. The period integral above is similar to (3.1), and thus satisfies a similar GKZ system to (3.3). The only difference appears in the scaling properties expressed by the linear operators $\mathcal{Z}_{i}$.

Proposition 4.2. 1) The period integral 4.2) satisfies

$$
\square_{l} \int_{C} \mathcal{U}(a)=0(l \in L), \quad \mathcal{Z}_{i}^{\prime} \int_{C} \mathcal{U}(a)=0(i=1,2),
$$

where the operators $\square_{l}$ and the lattice $L$ are the same as in (3.3), and $Z_{i}^{\prime}(i=1,2)$ are given by

$$
\left(\begin{array}{l}
\mathcal{Z}_{1}^{\prime} \\
\mathcal{Z}_{2}^{\prime}
\end{array}\right)=\left(\begin{array}{c}
\theta_{1}+\theta_{2}+\cdots+\theta_{\mu+2} \\
\theta_{2}+2 \theta_{3} \cdots+(\mu+1) \theta_{\mu+2}+1
\end{array}\right)
$$

2) The system (4.3) above is reducible of rank $\mu+1$ with its irreducible part of rank $\mu$. The $\mu$ independent solutions of the irreducible part are given by

$$
\beta_{0}(a)-\beta_{1}(a), \cdots, \beta_{\mu-1}(a)-\beta_{\mu}(a),
$$

where $\beta_{i}(a)$ 's are roots of $\psi(W)=a_{1}+a_{2} W+\cdots+a_{\mu+2} W^{\mu+1}=0$. 
Derivation of 1) above is straightforward, but it should be noted that K. Saito's system is replaced by a different but a simple GKZ system. Also, it is known that the numbers of independent solutions of a GKZ system is given by the volume of a relevant polytope GKZ1, which in our case $\operatorname{Vol}(\Sigma)=\mu+1$. One may verify that the system is reducible observing a factorization of a differential operator in our GKZ system when expressing $\square_{l}$ operators in an affine coordinate of $\mathcal{M}_{\operatorname{Sec}(\Sigma)}$. After the factorization, the reduced GKZ system has $\mu$ independent solutions as claimed. (It has been observed in [HKTY1] that the GKZ systems in mirror symmetry are often reducible in this way.) One can derive the $\mu$ independent solutions in 2) by evaluating the period integral (4.2) over the vanishing cycles (, see Appendix A). Or more directly, one may verify that the roots $\beta_{k}$ 's are solutions of (4.3) with one relation $\beta_{0}+\cdots+\beta_{\mu}=-\frac{a_{\mu+1}}{a_{\mu+2}}$ from the following property:

Proposition 4.3. The root $\beta(a)\left(=\beta_{0}(a), \cdots, \beta_{\mu}(a)\right)$ of $\psi(W)=0$ satisfies

$$
\frac{\partial \beta}{\partial a_{i}}=-\frac{\beta^{i-1}}{\psi^{\prime}(\beta)}, \quad \frac{\partial^{2} \beta}{\partial a_{i} \partial a_{j}}=\left.\frac{1}{\psi^{\prime}(\beta)} \frac{d}{d x}\left(\frac{x^{i+j-2}}{\psi^{\prime}(x)}\right)\right|_{x=\beta} .
$$

Proof. The first relation follows from the differentiation by $a_{i}$ of $\psi(\beta(a))=a_{1}+$ $a_{2} \beta(a)+\cdot+a_{\mu+2} \beta(a)^{\mu+1}=0$. For the second relation, we note the following;

$$
\frac{\partial \psi^{\prime}(\beta)}{\partial a_{j}}=(j-1) \beta^{j-2}+\psi^{\prime \prime}(\beta) \frac{\partial \beta}{\partial a_{j}}=(j-1) \beta^{j-2}-\frac{\psi^{\prime \prime}(\beta)}{\psi^{\prime}(\beta)} \beta^{j-1} .
$$

Then the second relation follows by differentiating the first equation with respect to $a_{i}$.

Now a proof of Proposition 4.2 is straightforward by the relations above. Here, since the arguments are similar, we present the solutions of the GKZ system (3.3) for the local mirror symmetry, which is the same as the GKZ system (4.3) for the primitive form except the scalings operators.

Proposition 4.4. 1) The independent solutions of the GKZ system 3.3) are given by

$$
1, \log \beta_{0}(a)-\log \beta_{1}(a), \cdots, \log \beta_{\mu-1}(a)-\log \beta_{\mu}(a) .
$$

2) These solutions, up to monodromy transformations, are related to the expansion $w\left(x ; \frac{J}{2 \pi i}\right)=1+\sum_{k=1}^{\mu} w_{k}(a) J_{k}$ near the large complex structure by

$$
2 \pi i w_{k}(x)=\log \beta_{k-1}(a)-\log \beta_{k}(a) \quad(k=1, \cdots, \mu) .
$$

Proof. 1) By the relations (4.4), it it straightforward to evaluate $\mathcal{Z}_{1} \log \beta$ and $\mathcal{Z}_{2} \log \beta$ as follows;

$$
\begin{aligned}
& \mathcal{Z}_{1} \log \beta=\frac{1}{\beta} \sum_{i=1}^{\mu+2} \theta_{a_{i}} \beta=-\frac{1}{\beta} \sum_{i=1}^{\mu+2} \frac{1}{\psi^{\prime}(\beta)} a_{i} \beta^{i-1}=-\frac{1}{\beta \psi^{\prime}(\beta)} \psi(\beta)=0, \\
& \mathcal{Z}_{2} \log \beta=\frac{1}{\beta} \sum_{i=1}^{\mu+2}(i-1) \theta_{a_{i}} \beta=-\frac{1}{\psi^{\prime}(\beta)} \sum_{i=1}^{\mu+2}(i-1) a_{i} \beta^{i-2}=-1 .
\end{aligned}
$$


S. HOSONO

Therefore the differences $\log \beta_{k}-\log \beta_{k-1}(k=1, . ., \mu)$ are annihilated by the linear operators $\mathcal{Z}_{1}, \mathcal{Z}_{2}$. As for the operators $\square_{l}(l \in L)$, we first note

$$
\frac{\partial^{2}}{\partial a_{i} \partial a_{j}} \log \beta=\frac{\partial^{2}}{\partial a_{k} \partial a_{l}} \log \beta \quad \text { if } i+j=k+l,
$$

which follows from

$$
\frac{\partial^{2}}{\partial a_{i} \partial a_{j}} \log \beta=-\frac{1}{\beta^{2}} \frac{\beta^{i+j-2}}{\left(\psi^{\prime}(\beta)\right)^{2}}+\left.\frac{1}{\beta} \frac{1}{\psi^{\prime}(\beta)} \frac{d}{d x}\left(\frac{x^{i+j-2}}{\psi^{\prime}(x)}\right)\right|_{x=\beta} .
$$

Since the entries of $l=\left(l_{1}, l_{2}, \ldots, l_{\mu+2}\right)$ satisfies $\sum_{i} l_{i}=0$ and $\sum_{i}(i-1) l_{i}=0$, we have $\sum_{i} i l_{i}^{+}=\sum_{i} i l_{i}^{-}$for $l_{i}^{ \pm}:=\frac{1}{2}\left(l_{i} \pm\left|l_{i}\right|\right)$. Now, by repeated use of (4.5), one may deduce

$$
\prod_{i}\left(\frac{\partial}{\partial a_{i}}\right)^{l_{i}^{+}} \log \beta=\prod_{i}\left(\frac{\partial}{\partial a_{i}}\right)^{l_{i}^{-}} \log \beta,
$$

which means $\square_{l} \log \beta=0$. Since the linear independence of $\log \beta_{k}-\log \beta_{k-1}$ ( $k=$ $1, . ., \mu)$ is clear, we obtain the claim including the trivial solution 1.

2) By definition, we have $2 \pi i w_{k}(x) \sim \log x_{k}$, where $x_{k}=\frac{a_{k} a_{k+2}}{a_{k+1}^{2}}$. Since, by extending the classical arguments due to Frobenius, it is straightforward to show that $w_{k}(x)$ 's are solutions of the GKZ hypergeometric system (3.3), we only need to connect these solutions to those in terms of roots $\beta_{k}$. For this, let us assume that the roots are given by $\beta_{k}=-\frac{1}{\lambda_{k}}$ and also the following relation,

$$
\psi(W)=a_{1}+a_{2} W+\cdots+a_{\mu+2} W^{\mu+1}=\prod_{k}\left(\lambda_{k} W+1\right) .
$$

Assume also that $\left|\lambda_{\mu}\right|<<\left|\lambda_{\mu-1}\right|<<\cdots<<\left|\lambda_{0}\right|$. Then from the relations between roots and the coefficients, it is easy to see

$$
\begin{gathered}
\log a_{1}=0, \log a_{2}=\log \lambda_{0}+A_{2}(\lambda), \log a_{3}=\log \left(\lambda_{0} \lambda_{1}\right)+A_{3}(\lambda), \cdots, \\
\log a_{\mu+2}=\log \left(\lambda_{0} \lambda_{1} \cdots \lambda_{\mu}\right)+A_{\mu+2}(\lambda),
\end{gathered}
$$

where $A_{k}(\lambda) \in \mathbf{C}\left\{\frac{\lambda_{1}}{\lambda_{0}}, \frac{\lambda_{2}}{\lambda_{1}}, \cdots, \frac{\lambda_{\mu}}{\lambda_{\mu-1}}\right\}$. Now we have

$$
\log x_{k}=\log \frac{a_{k} a_{k+2}}{a_{k+1}^{2}}=\log \lambda_{k}-\log \lambda_{k-1}+B_{k}(\lambda),
$$

with $B_{k}(\lambda):=A_{k}(\lambda)+A_{k+2}(\lambda)-2 A_{k+1}(\lambda)$, which entails the claimed relation since $2 \pi i w_{k}(x) \sim \log x_{k}$. (Note that if we invert the series relations $x_{k}=\frac{\lambda_{k}}{\lambda_{k-1}} e^{B_{k}(\lambda)}$ in $C\left\{\frac{\lambda_{1}}{\lambda_{0}}, \frac{\lambda_{2}}{\lambda_{1}}, \cdots, \frac{\lambda_{\mu}}{\lambda_{\mu-1}}\right\}$, then we obtain $\frac{\lambda_{k}}{\lambda_{k-1}}$ 's in terms of $x_{k}$ 's, and thus recover the series expansion of $2 \pi i w_{k}(x)$ by $2 \pi i w_{k}(x)=\log \lambda_{k}-\log \lambda_{k-1}=\log x_{k}-B_{k}(\lambda)$.)

The above form of the solutions may be connected to the piecewise linear functions on the fan $\Sigma[\mathrm{Ba3}$. Since we have established a relation of the GKZ system (3.3) of $\mathbf{C}^{2 / \mathbf{Z}_{\mu+1}}$ to K.Saito's system of the primitive form, for which integral monodromy property is well studied (see Proposition 4.1), it is clear that we have the uniformization property of the mirror map stated in Proposition 3.2 


\section{Central charge formula and G-Hilb}

In the last two sections, we have looked at the local mirror symmetry of $\widehat{\mathbf{C}^{2} / \mathbf{Z}_{\mu+1}}$ paying our attentions to the monodromy property of the associated GKZ system. Here we come back to our claim for the central charge formula (2.4) in this case.

Let us first recall that the non-compact Calabi-Yau manifold $X=\widehat{\mathbf{C}^{2 / \mathbf{Z}_{\mu+1}}}$ is given as the Hilbert scheme of points on $\mathbf{C}^{2}$, G-Hilb $\mathbf{C}^{2}$. Here G-Hilb $\mathbf{C}^{n}$ is defined for a finite subgroup $G \subset S L(n, \mathbf{C})$ and consists of zero dimensional subschemes $Z$ in $\mathbf{C}^{n}$ of length equal $|G|$ such that $G$ acts on $Z$ and $H^{0}\left(\mathcal{O}_{Z}\right)$ is the regular representation of $G$. The following results are due to Gonzalez-Sprinberg and Verdier GV] for $n=2$ and their generalizations to $n=3$ have been done in [Na] [N] [BKR]. Here, we summarize the relevant results for our cases where $G \subset S U(n, \mathbf{C})$ is a finite abelian subgroup:

(i) The K-group $K(X)$ of algebraic vector bundles on $X$ are generated by the socalled tautological bundles $\mathcal{F}_{0}, \mathcal{F}_{1}, \cdots, \mathcal{F}_{d}(d=|G|-1)$, where the subscripts refer to the one-dimensional representations of $G$ with 0 for the trivial representation.

(ii) Let $K^{c}(X)$ be the K-group of the complexes of algebraic vector bundles which are exact off $\pi^{-1}(0)$ where $\pi: X \rightarrow \mathbf{C}^{n} / G$. Then there exists a complete pairing $K^{c}(X) \times K(X) \rightarrow \mathbf{Z}$, and the dual basis $S_{0}, S_{1}, \cdots, S_{d}$ of $K^{c}(X)$ satisfying

$$
\left\langle\operatorname{ch}\left(S_{i}\right), \operatorname{ch}\left(\mathcal{F}_{j}\right)\right\rangle:=\int_{X} \operatorname{ch}\left(S_{i}\right) \operatorname{ch}\left(\mathcal{F}_{j}\right) \operatorname{Todd}_{X}=\delta_{i j} .
$$

(iii) The dual basis $S_{k}(k=0, \cdots, d)$ defines a symmetric form for $n=2$ (, a symplectic form for $n=3$,) on $K^{c}(X)$ by

$$
\chi\left(S_{i}, S_{j}\right):=\int_{X} \operatorname{ch}\left(S_{i}^{\vee}\right) \operatorname{ch}\left(S_{j}\right) \operatorname{Todd}_{X} \quad(0 \leq i, j \leq d) .
$$

In two dimensional case with $G=\mathbf{Z}_{\mu+1}, S_{k}$ 's for $k \neq 0$ have a simple forms. They are given by $S_{k}=\mathcal{O}_{C_{k}}(-1)$ with $C_{k} \cong \mathbf{P}^{1}$ being the exceptional curves. Including $S_{0}$, they satisfy $\chi\left(S_{i}, S_{j}\right)=-\hat{\mathcal{C}}_{i j} \quad(0 \leq i, j \leq d)$ where $\hat{\mathcal{C}}_{i j}$ is the extended Cartan matrix of the root system of type $A_{\mu}$.

Now with these properties about the geometry of $X=\mathbf{C}^{2 / \mathbf{Z}_{\mu+1}}$, let us recall that our Conjecture 2.2

$$
w\left(x_{1}, \cdots, x_{r} ; \frac{J_{1}}{2 \pi i}, \cdots, \frac{J_{r}}{2 \pi i}\right)=\sum_{i, j} \int_{\operatorname{mir}\left(E_{i}\right)} \Omega\left(Y_{x}\right) \chi^{i j} \operatorname{ch}\left(E_{j}^{\vee}\right) .
$$

One should note that this is a conjecture for $X$ a compact Calabi-Yau manifold. It is rather clear how to modify this relation for our non-compact $X$ if we notice that the expression $\sum_{j} \chi^{i j} \operatorname{ch}\left(E_{j}^{\vee}\right)$ satisfies $\int_{X} \operatorname{ch}\left(E_{k}\right) \sum_{j} \chi^{i j} \operatorname{ch}\left(E_{j}^{\vee}\right) \operatorname{Tod} d_{X}=\delta_{k}^{i}$, i.e., provides a dual base to $\operatorname{ch}\left(E_{k}\right)$. From this, we claim that the central charge formula for $X=\mathbf{C}^{2 / \mathbf{Z}_{\mu+1}}$ is given by

$$
w\left(x_{1}, \cdots, x_{\mu} ; \frac{J_{1}}{2 \pi i}, \cdots, \frac{J_{\mu}}{2 \pi i}\right)=\sum_{k} \int_{\operatorname{mir}\left(S_{k}\right)} \Omega\left(Y_{x}\right) \operatorname{ch}\left(\mathcal{F}_{k}\right),
$$


where the holomorphic two form $\Omega\left(Y_{x}\right)$ should be understood as a holomorphic twoform associated to the integrand of (3.1) which is similar to the primitive form in (4.2). We will state the corresponding claim in Conjecture 6.3 for three dimensional cases.

Now, since the tautological bundles satisfy

$$
\int_{C_{j}} c_{1}\left(\mathcal{F}_{k}\right)=\delta_{j k} \quad(1 \leq j, k \leq \mu)
$$

for the exceptional curves $C_{j}(1 \leq j \leq \mu)$, we have $c_{1}\left(\mathcal{F}_{k}\right)=J_{k}(k \geq 1)$ for our generators $J_{k}$ of $H^{2}(X, \mathbf{Z})$. Therefore we have

$$
w\left(x ; \frac{J}{2 \pi i}\right)=1+\sum_{k} w_{k}(x) J_{k}=\left(1-\sum_{k=1}^{\mu} w_{k}(x)\right) \operatorname{ch}\left(\mathcal{F}_{0}\right)+\sum_{k=1}^{\mu} w_{k}(x) \operatorname{ch}\left(\mathcal{F}_{k}\right),
$$

where we use $\operatorname{ch}\left(\mathcal{F}_{k}\right)=1+c_{1}\left(\mathcal{F}_{k}\right)=1+J_{k}$. From this, one may read the central charge of the sheaf $S_{k}=\mathcal{O}_{C_{k}}(-1)(k \neq 0)$ as

$$
Z_{t}\left(S_{k}\right)=\int_{\operatorname{mir}\left(S_{k}\right)} \Omega\left(Y_{x}\right)=w_{k}(x)=\frac{1}{2 \pi i}\left(\log \beta_{k-1}(a)-\log \beta_{k}(a)\right) .
$$

In Appendix A, we obtain the above central charge as a period integral over a vanishing cycle. Therefore, we have obtained a refinement of the mirror symmetry sketched in (3-1) that the mirror image $\operatorname{mir}\left(S_{k}\right)$ of the sheaf $S_{k}=\mathcal{O}_{C_{k}}(-1)$ is a vanishing cycle whose period is given by $Z_{t}\left(S_{k}\right)$ above.

Similar relation holds also for $S_{0}$ with its central charge $1-\sum_{k} w_{k}(x)$. However, since $S_{0}$ is not a sheaf but a complex of sheaves (see [IN]), we replace it with the skyscraper sheaf $\mathcal{O}_{p}$ supported on a point $p$. Then the sheaves $\mathcal{O}_{p}, \mathcal{O}_{C_{k}}(-1)(k=$ $1, \cdots, \mu)$ make another basis of $K^{c}(X)$ with relations

$$
\mathcal{O}_{p}=S_{0}+S_{1}+\cdots+S_{\mu}, \mathcal{O}_{C_{k}}(-1)=S_{k}(k=1, \cdots, \mu),
$$

where we use $\left\langle\mathcal{O}_{p}, \mathcal{F}_{j}\right\rangle=1(j=0, \cdots, \mu)$ to derive the first equality. In (6-2), this basis of $K^{c}(X)$ will be generalized as a symplectic D-brane basis to three dimensional cases. Now, for the sheaf $\mathcal{O}_{p}$, we have

$$
Z_{t}\left(\mathcal{O}_{p}\right)=\int_{\operatorname{mir}\left(\mathcal{O}_{p}\right)} \Omega\left(Y_{x}\right) \equiv 1
$$

This is identified with the period integral over a cycle $T_{0}$ whose topology is $S^{1} \times S^{1}$, see Appendix A.

Finally, we note that the sheaves $S_{k}(k \neq 0)$ are spherical and thus define selfequivalences of $D^{b}(\operatorname{Coh}(X))$, the Seidel-Thomas twists summarized in section 2. In [ST, Proposition 3.19], it is shown that these spherical objects, which form the socalled $\left(A_{\mu}\right)$-configuration, generate a weak braid group action on $D^{b}(\operatorname{Coh}(X))$. This braid group action should be mirrored to the corresponding Dehn twists (Picard-Lefschetz transformations) in the symplectic side. Our formula (5.4) explicitly shows this mirror correspondence as the linear transformations on the central charges. 


\section{Local mirror symmetry II $-X=\widehat{\mathbf{C}^{3} / G}$}

In the two dimensional cases, the local mirror symmetry is rather clear from the hyperkähler rotation as summarized in (3-1), and our results in the previous sections are consistent with those arguments from the hyperkähler rotation. In this section, we will argue that our central charge formula for a crepant resolution of the three dimensional singularity $\mathbf{C}^{3} / G$ with a finite abelian group $G \subset S U(3, \mathbf{C})$. The crepant resolution we use is the so-called $G$-Hilb $\mathbf{C}^{3}$ whose definition has been given in the previous section. Examples of GKZ hypergeometric system in this situation have been studied also in dOFS.

(6-1) Period integrals and GKZ systems. As above, let us consider a non-compact Calabi-Yau manifold $X=G$-Hilb $\mathbf{C}^{3}$ with a finite, abelian group $G \subset S U(3, \mathbf{C})$. For $G$ being abelian, $X$ is given as a toric resolution of a singularity $\mathbf{C}^{3} / G$ as follows: Let us first denote by $M_{G}$ the lattice corresponding to the invariants $\mathbf{C}\left[x^{ \pm}, y^{ \pm}, z^{ \pm}\right]^{G}$, and its dual lattice by $N_{G}$. Then the invariant monomials in $\mathbf{C}[x, y, z]^{G}$ defines a cone in $M_{G} \otimes \mathbf{R}$, with its dual cone $\Sigma_{G}^{0}$ in $N_{G} \otimes \mathbf{R}$. The toric (Calabi-Yau) resolution which corresponds to $G$-Hilb $\mathbf{C}^{3}$ is given by a fan obtained by subdividing the cone $\Sigma_{G}^{0}$, which we will denote by $\Sigma_{G}$ hereafter. (See [Re] for detailed construction of the fan $\Sigma_{G}$.) We will denote by $\nu_{1}, \nu_{2}, \cdots, \nu_{r+3}$ the integral generators of the one-dimensional cones in the fan $\Sigma_{G}$. Note that, since the resolution is crepant, these generators satisfy

$$
\left(m, \nu_{k}\right)=1 \quad(k=1, \cdots, r+3),
$$

with some $m \in M_{G}$, where $():, M_{G} \times N_{G} \rightarrow \mathbf{Z}$ is the dual pairing.

To describe the mirror configuration of $X$, let us fix an isomorphism $\varphi: N_{G} \simeq \mathbf{Z}^{3}$ satisfying $\varphi\left(\nu_{k}\right)=\left(1, \bar{\nu}_{k}\right)$. Consider the defining equation

$$
F\left(a ; U, V, W_{1}, W_{2}\right)=U^{2}+V^{2}+\sum_{k} a_{k} W^{\bar{\nu}_{k}},
$$

then the mirror configuration of $X$ claimed in [CKYZ] [HIV] is the hypersurface $Y=\left(F\left(a ; U, V, W_{1}, W_{2}\right)=0\right) \subset \mathbf{C}^{2} \times\left(\mathbf{C}^{*}\right)^{2}$. Note that $Y$ does not depend on the choice of the isomorphism $\varphi: N_{G} \stackrel{\sim}{\rightarrow} \mathbf{Z}^{3}$ with the described property, since $W_{1}, W_{2}$ are considered in $\mathbf{C}^{*}$ and negative powers of them are allowed. One important criterion for the mirror configuration $Y$ is the fact that we can extract the right Gromov-Witten invariants for $X$ from the "period integrals" of $Y$ (cf. CKYZ).

Definition 6.1. For the mirror configuration $Y$ defined above, we define the "period integral" of a cycle $\gamma$ by

$$
\Pi_{\gamma}(a)=\int_{\gamma} \frac{1}{U^{2}+V^{2}+\sum_{k} a_{k} W^{\bar{\nu}_{k}}} d U d V \frac{d W_{1}}{W_{1}} \frac{d W_{2}}{W_{2}},
$$

for a cycle $\gamma \in H_{4}\left(\mathbf{C}^{2} \times\left(\mathbf{C}^{*}\right)^{2} \backslash Y, \mathbf{Z}\right)$.

In (6-3), we will describe how one can determine the Gromov-Witten invariants from our cohomology-valued hypergeometric series. Here, as in the two dimensional cases, we see that the following GKZ system is satisfied by the period integral: 
Proposition 6.2. Let $L$ be the lattice of relations defined by

$$
L=\left\{\left(l_{1}, \cdots, l_{r+3}\right) \in \mathbf{Z}^{r+3} \mid \sum_{k} l_{k} \nu_{k}=\overrightarrow{0}\right\} .
$$

Then the period integral (6.2) satisfies the following set of differential equations:

$$
\square_{l} \Pi_{\gamma}(a)=0(l \in L), \quad \mathcal{Z}_{i} \Pi_{\gamma}(a)=0(i=1,2,3),
$$

where

$$
\square_{l}=\left(\frac{\partial}{\partial a}\right)^{l^{+}}-\left(\frac{\partial}{\partial a}\right)^{l^{-}} \quad,\left(\begin{array}{l}
\mathcal{Z}_{1} \\
\mathcal{Z}_{2} \\
\mathcal{Z}_{3}
\end{array}\right)=\left(\begin{array}{c}
\theta_{1}+\theta_{2}+\cdots+\theta_{r+3} \\
\sum_{k} \bar{\nu}_{k, 2} \theta_{k} \\
\sum_{k} \bar{\nu}_{k, 3} \theta_{k}
\end{array}\right)
$$

with $l=l^{+}-l^{-}\left(l_{i}^{ \pm}:=\frac{1}{2}\left(l_{i} \pm\left|l_{i}\right|\right)\right)$ and $\theta_{k}=a_{k} \frac{\partial}{\partial a_{k}} . \bar{\nu}_{k, i}$ represents the $i$-th component of the vector $\varphi\left(\nu_{k}\right)=\left(1, \bar{\nu}_{k}\right)$ with the isomorphism $\varphi: N_{G} \stackrel{\sim}{\rightarrow} \mathbf{Z}^{3}$ fixed above.

Remark. As we have shown in section 4, by a slight modification of the period integral, we can connect (6.2) to the K. Saito's primitive form of a three dimensional singularirity. The primitive form satisfies the same GKZ hypergeometric system but with a different scaling property, i.e., $\mathcal{Z}_{i}(i=2,3)$ being replaced by $\mathcal{Z}_{i}^{\prime}:=\mathcal{Z}_{i}+1(i=$ $2,3)$. One may observe for several examples that, when the singularity of $\mathbf{C}^{3} / G$ is isolated, the resulting GKZ system for the primitive form is reducible and its irreducible part shows a slightly different degeneration at 'infinity', i.e. at the large complex structure limit (, see Appendix B).

(6-2) Central charge formula and symplectic forms. The cohomologyvalued hypergeometric series for the GKZ system (6.3) are defined by constructing the secondary fan $\operatorname{Sec}\left(\Sigma_{G}\right)$. As described for an example in (3-3) and Fig.1, the secondary fan is constructed combinatorially from the secondary polytope in (a translate of) $L_{\mathbf{R}}$. We refer [HLY], [Hos, [Sti] and references therein for details of the construction in our context of mirror symmetry.

Let us denote by $l^{(1)}, \cdots, l^{(r)}$ the integral generators of $L$ which represents the large complex structure limit. We denote the affine coordinate by $x_{k}=a^{l^{(k)}}(k=$ $1, \cdots, r)$. Then, we have cohomology-valued hypergeometric series

$$
w\left(x_{1}, \cdot \cdot, x_{r} ; \frac{J_{1}}{2 \pi i}, \cdot \cdot \frac{J_{r}}{2 \pi i}\right)=\left.\sum_{m \in \mathbf{Z}_{\geq 0}^{s}} c(m+\rho) x^{m+\rho}\right|_{\rho=\frac{J}{2 \pi i}},
$$

where $c(m):=c\left(m_{1}, \cdots, m_{r}\right)$ is defined by

$$
c\left(m_{1}, \cdots, m_{r}\right):=\frac{1}{\prod_{i=1, \ldots, r+3} \Gamma\left(1+\sum_{k} m_{k} l_{i}^{(k)}\right)},
$$

and $J_{1}, \cdots, J_{r} \in H^{2}(X, \mathbf{Z})$ are dual (semi-ample) generators to $l^{(1)}, \cdots, l^{(r)}$ (, see [Sect.1, Hos] for a quick review of the construction). It is found in HKTY2 for $X$ compact Calabi-Yau hypersurfaces (see also Sti]), and used in [CKYZ for noncompact cases that the above cohomology-valued hypergeometric series generate the solutions of the GKZ system (6.3) when expanded in $H^{\text {even }}(X, \mathbf{Q})$. If we recall the integral structure of $K(X)$ and also the natural symplectic structure in $K^{c}(X)$ which are summarized in section 4 , we come to the following conjecture: 
Conjecture 6.3. 1) Consider the expansion of the cohomology-valued hypergeometric series 6.4) with respect to the basis $\operatorname{ch}\left(\mathcal{F}_{0}\right), \cdots, \operatorname{ch}\left(\mathcal{F}_{d}\right)$ of $H^{\text {even }}(X, \mathbf{Q})$; $w\left(x ; \frac{J}{2 \pi i}\right)=\sum_{k} f_{k}(x) \operatorname{ch}\left(\mathcal{F}_{k}\right)$. Then the coefficient hypergeometric series $f_{k}(x)$ may be identified with the period integrals over the cycles $\operatorname{mir}\left(S_{k}\right)$, i.e., we have

$$
w\left(x_{1}, \cdots, x_{r} ; \frac{J_{1}}{2 \pi i}, \cdots, \frac{J_{r}}{2 \pi i}\right)=\sum_{k} \int_{\operatorname{mir}\left(S_{k}\right)} \Omega\left(Y_{x}\right) \operatorname{ch}\left(\mathcal{F}_{k}\right),
$$

where mir : $K^{c}(X) \stackrel{\sim}{\rightarrow} H_{3}(Y, \mathbf{Z})$ is the (homological) mirror map and $\Omega\left(Y_{x}\right)=$ $\operatorname{Res}_{F=0}\left(\frac{1}{F\left(U, V, W_{1}, W_{2}\right)} d U d V \frac{d W_{1}}{W_{1}} \frac{d W_{2}}{W_{2}}\right)$.

2) The monodromy of the coefficient hypergeometric series $f_{k}(x)\left(=\int_{\operatorname{mir}\left(S_{k}\right)} \Omega\left(Y_{x}\right)\right)$ is integral and symplectic with respect to the symplectic form;

$$
\chi\left(S_{i}, S_{j}\right)=\int_{X} \operatorname{ch}\left(S_{i}^{\vee}\right) \operatorname{ch}\left(S_{j}\right) \operatorname{Todd}_{X} \quad(0 \leq i, j \leq d=|G|-1) .
$$

3) Using the cohomology-valued hypergeometric series, the central charge of $F \in$ $K^{c}(X)$ is given by $Z_{t}(F)=\int_{X} \operatorname{ch}(F) w\left(x ; \frac{J}{2 \pi i}\right) \operatorname{Todd}_{X}$.

In section 4 and section 5 , we have verified explicitly the corresponding conjecture for $X=\widehat{\mathbf{C}^{2} / \mathbf{Z}_{\mu+1}}$ by connecting our period integral to that of the primitive form in singularity theory. For three dimensions, however, little is known about the explicit form of the period integral of the primitive form. In the following sections, we will test our conjecture for the cases, $G=\mathbf{Z}_{3}, \mathbf{Z}_{5}$. Especially, for the former case, we will evaluate the period integrals of the primitive form, and connect them to the periods in mirror symmetry (, see Appendix A). In this case, we will be able to see the mirror correspondence mir : $K^{c}(X) \stackrel{\sim}{\rightarrow} H_{3}(Y, \mathbf{Z})$ from our Conjecture 6.3 which generalizes the correspondence between the spherical objects and the vanishing cycles presented in section 5 .

As another aspect of our conjecture, we will define the so-called prepotential which gives predictions of Gromov-Witten invariants on the geometry $X$. When $X$ is compact, the existence of the prepotential (special Kähler geometry) is ensured by a canonical symplectic basis of $H_{3}(Y, \mathbf{Z})$ and also by the property called Griffiths transversality St. Correspondingly, in our non-compact cases, we have the symplectic structure on $H_{3}(Y, \mathbf{Z})$ given by $\left(\chi\left(S_{i}, S_{j}\right)\right)$ under the homological mirror map, mir : $K^{c}(X) \stackrel{\sim}{\rightarrow} H_{3}(Y, \mathbf{Z})$. We will find that our cohomology-valued hypergeometric series combined with the symplectic structure suffices to present a closed formula of the prepotential.

(6-3) Prepotential and a symplectic $D$-brane basis. In string theory, the so-called D-branes play important roles for duality symmetries such as mirror symmetry. In our non-compact Calabi-Yau manifolds, roughly speaking, the D-branes are elements in $K^{c}(X)$ or more precisely elements in the category $D_{c}^{b}(C o h(X))$ of compact support. In what follows, we will work on $K^{c}(X)\left(\right.$ or $H_{c}^{\text {even }}(X, \mathbf{Q})$ taking the Chern character). In our case, it is easy to see that there are natural D-branes like $\mathcal{O}_{p}$, the skyscraper sheaf, $\mathcal{O}_{C}$, the torsion sheaf supported on a curve $C$, and $\mathcal{O}_{D}$, that supported on an exceptional divisor $D$. Depending on the dimensions of the support, these sheaves are called $D 0-, D 2-, D 4$-branes, respectively, see [Do] and references therein. We will connect the bases $S_{i}$ 's to these $D$-branes. 
(1) Symplectic D-brane basis and its dual: First, let us recall that we have introduced the (semi-ample) generators $J_{1}, \cdots, J_{r}$ of $H^{2}(X, \mathbf{Z})$ when writing down our cohomology-valued hypergeometric series. Assume that these generators measure the volume of the respective curves $C_{1}, \cdots, C_{r}$, i.e.,

$$
\int_{C_{i}} J_{k}=\delta_{i k}
$$

Assume also that the exceptional divisors $D_{1}, \cdots, D_{s}$ provide a basis of $H_{4}(X, \mathbf{Z})$. Then in such cases, we seek a basis of $K^{c}(X)$ of the form,

$$
\left\{\mathcal{B}_{I}\right\}:=\left\{\mathcal{O}_{p} ; \mathcal{O}_{C_{1}}\left(-J_{1}\right), \cdots, \mathcal{O}_{C_{r}}\left(-J_{r}\right) ; \mathcal{O}_{D_{1}}\left(\mathcal{L}_{1}\right), \cdots, \mathcal{O}_{D_{s}}\left(\mathcal{L}_{s}\right)\right\}
$$

where $\mathcal{O}_{C}(-J)=\mathcal{O}_{C} \otimes \mathcal{O}_{X}(-J)$ and $\mathcal{O}_{D_{k}}\left(\mathcal{L}_{k}\right):=\mathcal{O}_{D} \otimes \mathcal{L}_{k}$ with $\mathcal{L}_{k}=\mathcal{O}_{X}\left(-a_{k, 1} J_{1}-\right.$ $\left.\cdots-a_{k, r} J_{r}\right)$. Here we fix the integers $a_{k, 1}, \ldots, a_{k, r}$ requiring

$$
\chi\left(\mathcal{O}_{D_{j}}\left(\mathcal{L}_{j}\right), \mathcal{O}_{D_{k}}\left(\mathcal{L}_{k}\right)\right)=0
$$

for $j, k=1, \cdots, s$. The integers $a_{k, 1}, \ldots, a_{k, r}$ satisfying the above requirement are not unique in general. However the reason of our requirement (6.7) will become clear soon when we present a closed formula of the prepotential. Hereafter, we will call a basis $\left\{\mathcal{B}_{I}\right\}$ satisfying the requirement as a symplectic D-brane basis of $K^{c}(X)$.

Now let us consider the dual basis of $K(X)$ to the basis $\left\{\mathcal{B}_{I}\right\}$ (6.6) under the paring (5.1). Taking $c h: K(X) \rightarrow H^{\text {even }}(X, \mathbf{Q})$, we construct (charges) $\left\{Q_{J}\right\}:=$ $\left\{Q_{0} ; Q_{2}^{1}, . ., Q_{2}^{r} ; Q_{4}^{1}, . ., Q_{4}^{s}\right\}$ which satisfies

$$
\int_{X} \operatorname{ch}\left(\mathcal{B}_{I}\right) Q_{J} \operatorname{Todd}_{X}=\delta_{I J}
$$

We note that, due to the support property of the D-brane basis (6.6) and the above relation, the base $Q_{0}$ starts from the degree zero element 1 to higher degree terms. Similarly $Q_{2}^{a}$ starts from $J_{a}$ to higher degree terms, and $Q_{4}^{b}$ is written by a linear combination of the degree four elements of $H^{\text {even }}(X, \mathbf{Q})$. The set of charges $\left\{Q_{J}\right\}$ provides a basis of $H^{\text {even }}(X, \mathbf{Q})$.

(2) Expansion of $w\left(x ; \frac{J}{2 \pi i}\right)$ and the prepotential: Now we expand our cohomologyvalued hypergeometric series using the basis $\left\{Q_{J}\right\}$ of $H^{\text {even }}(X, \mathbf{Q})$;

$$
w\left(x ; \frac{J}{2 \pi i}\right)=w_{0}(x) Q_{0}+\sum_{a=1}^{r} w_{a}^{(1)}(x) Q_{2}^{a}+\sum_{b=1}^{s} w_{b}^{(2)}(x) Q_{4}^{b} .
$$

Then our conjecture says that the coefficient hypergeometric series $\left\{w_{0}(x), w_{a}^{(1)}(x)\right.$, $\left.w_{b}^{(2)}(x)\right\}$ represents the central charges of the sheaves $\left\{\mathcal{B}_{I}\right\}$ in 6.6 and whose monodromy matrices are integral and symplectic with respect to the symplectic form

$$
\left(\chi\left(\mathcal{B}_{I}, \mathcal{B}_{J}\right)\right)=\left(\begin{array}{ccc}
0 & 0 & 0 \\
0 & 0 & C_{a b} \\
0 & -C_{a b} & 0
\end{array}\right),
$$

where we set $C_{a b}=\chi\left(\mathcal{O}_{C_{a}}\left(-J_{a}\right), \mathcal{O}_{D_{b}}\left(\mathcal{L}_{b}\right)\right)$ and used the fact $\chi\left(\mathcal{O}_{p}, \mathcal{B}_{I}\right)=0$ for sheaves $\mathcal{B}_{I}$ of compact support. The above form should be contrasted to the canonical symplectic form on $H_{3}(Y, \mathbf{Z})$ for the compact (mirror) Calabi-Yau manifolds, by which we define the special Kähler geometry on the moduli spaces(, see e.g. 
St]). In fact, we may define the prepotential for local mirror symmetry given in CKYZ in terms of the symplectic form (6.8).

For simplicity, let us assume $\operatorname{dim} H_{c}^{2}(X, \mathbf{Q})=\operatorname{dim} H_{c}^{4}(X, \mathbf{Q})($, i.e. $r=s)$, and thus $\left(C_{a b}\right)$ is a square matrix. Also we assume $\operatorname{det}\left(C_{a b}\right) \neq 0$. In this case, inverting the matrix $\left(C_{a b}\right)$, we may define the 'symplectic duals' of $\mathcal{O}_{C_{1}}\left(-J_{1}\right), \cdots, \mathcal{O}_{C_{r}}\left(-J_{r}\right)$ by $\sum_{b} C^{a b} \mathcal{O}_{D_{b}}\left(\mathcal{L}_{b}\right)(a=1, \cdots, r)$ where $\left(C^{a b}\right):=\left(C_{a b}\right)^{-1}$. We should note that these symplectic duals are not in $K^{c}(X)$ in general, but are elements in $K^{c}(X) \otimes \mathbf{Q}$. Correspondingly we consider the symplectic duals of the mirror cycles $\operatorname{mir}\left(\mathcal{O}_{C_{a}}\left(-J_{a}\right)\right)$ by $\sum_{b} C^{a b} \operatorname{mir}\left(\mathcal{O}_{D_{b}}\left(\mathcal{L}_{b}\right)\right)$. From the period integrals of these cycles, we define the prepotential $F(t)$ by the special Kähler geometry relation;

$$
\left(1, t_{a}, \frac{\partial F}{\partial t_{a}}\right)=\left(1, w_{a}^{(1)}(x), \sum_{b} C^{a b} w_{b}^{(2)}(x)\right)
$$

where we use the fact $w^{(0)}(x)=Z\left(\mathcal{O}_{p}\right) \equiv 1$ for local mirror symmetry of $X=\widehat{\mathbf{C}^{3} / G}$ (cf. section 5). Integrating this special Kähler geometry relation, we obtain the prepotential, which gives the right predictions for Gromov-Witten invariants(, see CKYZ for examples).

For general $G$, the matrix $\left(C_{a b}\right)$ may not be square. Even if it is square it might be singular. In such cases, some of the sheaves $\mathcal{O}_{C_{a}}\left(-J_{a}\right)$ do not have its symplectic duals. However the special Kähler relation (6.9) still makes sense under suitable modifications.

(6-4) Examples. Here we will present two examples to verify Conjecture 6.3 Some detailed physical analyses of D-branes and $\Pi$ stability on the first example may be found in [DFR] (, see also [Ma Do and references therein). The hypergeometric series (with a different definition) of the second example are also studied in dOFS (see also $\mathrm{MR}]$ ).

Example (1): As the first example, we consider the case $X=\widehat{\mathbf{C}^{3} / \mathbf{Z}_{3}}$ with the group action specified by the weights $\frac{1}{3}(1,1,1)$. The toric resolution is the same as the $G$-Hilb and may be identified with the total space of the canonical bundle $K_{\mathbf{P}^{2}}$. The cohomology $H^{\text {even }}(X, \mathbf{Q})$ is generated by $1, J, J^{2}$ where $J$ is the class dual to a line $C$ in $\mathbf{P}^{2}$. As shown in the toric diagram in Fig.3, the toric divisor $D$ represents the section $\mathbf{P}^{2}$. The construction of the secondary polytope is easy, and we find $l^{(1)}=$ $(1,1,1,-3)$ for the local parameter $x=a^{l^{(1)}}$ of the GKZ hypergeometric system. With these data, we may write down the cohomology-valued hypergeometric series,

$$
w\left(x ; \frac{J}{2 \pi i}\right)=\left.\sum_{n=0}^{\infty} \frac{1}{\Gamma(1+n+\rho)^{3} \Gamma(1-3(n+\rho))} x^{n+\rho}\right|_{\rho=\frac{J}{2 \pi i}} .
$$

The Taylor series expansion with respect to $\rho$ and the ring structure of $H^{\text {even }}(X, \mathbf{Q})$ define the hypergeometric series of our interest. As conjectured in Conjecture 6.3 the integral and symplectic structure of the hypergeometric series comes from $K(X)$ and $K^{c}(X)$, respectively. 
The tautological line bundles make an integral basis of $K(X)$ and, following Re] $[\mathrm{Cr}$, they are determined easily as

$$
\mathcal{F}_{0}=\mathcal{O}_{X}, \mathcal{F}_{1}=\mathcal{O}_{X}(J), \mathcal{F}_{2}=\mathcal{O}_{X}(2 J) .
$$

We can determine the dual basis $S_{k}$ following IN]. Here, instead, we fix the Dbrane basis $\left\{\mathcal{B}_{I}\right\}$ to $\left\{\mathcal{O}_{p}, \mathcal{O}_{C}(-1), \mathcal{O}_{D}(-2)\right\}$, where $\mathcal{O}_{C}(-1):=\mathcal{O}_{C} \otimes \mathcal{O}_{X}(-J)$ and $\mathcal{O}_{D}(-2):=\mathcal{O}_{D} \otimes \mathcal{O}_{X}(-2 J)$. The relations to the bases $S_{k}$ are easily worked out to be

$$
\mathcal{O}_{p}=S_{0}+S_{1}+S_{2}, \quad \mathcal{O}_{C}(-1)=S_{1}+S_{2}, \mathcal{O}_{D}(-2)=S_{2},
$$

and also the symplectic form (6.8) becomes to

$$
\left(\chi\left(\mathcal{B}_{I}, \mathcal{B}_{J}\right)\right)=\left(\begin{array}{ccc}
0 & 0 & 0 \\
0 & 0 & -3 \\
0 & 3 & 0
\end{array}\right) .
$$

From the relations in (6.11), we find a basis $\left\{\mathcal{F}_{0}, \mathcal{F}_{1}-\mathcal{F}_{0}, \mathcal{F}_{0}-2 \mathcal{F}_{1}+\mathcal{F}_{2}\right\}$ of $K(X)$, which are dual to $\left\{\mathcal{O}_{p}, \mathcal{O}_{C}(-1), \mathcal{O}_{D}(-2)\right\}$. Taking ch: $K(X) \rightarrow H^{\text {even }}(X, \mathbf{Q})$, we then finally come to a basis $\left\{Q_{0}, Q_{2}, Q_{4}\right\}$ of $H^{\text {even }}(X, \mathbf{Q})$,

$$
Q_{0}=\operatorname{ch}\left(\mathcal{F}_{0}\right), Q_{2}=\operatorname{ch}\left(\mathcal{F}_{1}\right)-\operatorname{ch}\left(\mathcal{F}_{0}\right), Q_{0}=\operatorname{ch}\left(\mathcal{F}_{2}\right)-2 \operatorname{ch}\left(\mathcal{F}_{1}\right)+\operatorname{ch}\left(\mathcal{F}_{0}\right),
$$

by which we expand our cohomology-valued hypergeometric series;

$$
w\left(x ; \frac{J}{2 \pi i}\right)=w^{(0)}(x) Q_{0}+w^{(1)}(x) Q_{2}+w^{(2)}(x) Q_{4} .
$$

For convenience, we write explicitly the coefficient hypergeometric series;

$$
w^{(0)}(x)=1, w^{(1)}(x)=\partial_{\tilde{\rho}} w(x), w^{(2)}(x)=\frac{1}{2} \partial_{\tilde{\rho}}^{2} w(x)-\frac{1}{2} \partial_{\tilde{\rho}} w(x),
$$

where $\partial_{\tilde{\rho}}:=\frac{1}{2 \pi i} \frac{\partial}{\partial \rho}$.

Our Conjecture 6.3 predicts that the coefficient hypergeometric series $w^{(0)}(x)$, $w^{(1)}(x), w^{(2)}(x)$ thus obtained have integral and symplectic monodromy properties with respect to the symplectic form (6.12). We verify these properties in Appendix A. Moreover our Conjecture 6.3 predicts that these hypergeometric series represents period integrals over the respective mirror cycles of $\mathcal{O}_{p}, \mathcal{O}_{C}(-1), \mathcal{O}_{D}(-2)$ under mir : $K^{c}(X) \stackrel{\sim}{\rightarrow} H_{3}(Y, \mathbf{Z})$, i.e.,

$$
\begin{aligned}
& w^{(0)}(x)=\int_{\operatorname{mir}\left(\mathcal{O}_{p}\right)} \Omega\left(Y_{x}\right), \\
& w^{(1)}(x)=\int_{\operatorname{mir}\left(\mathcal{O}_{C}(-1)\right)} \Omega\left(Y_{x}\right), w^{(2)}(x)=\int_{\operatorname{mir}\left(\mathcal{O}_{D}(-2)\right)} \Omega\left(Y_{x}\right),
\end{aligned}
$$

where $Y$ is the mirror hypersurface $F\left(U, V, W_{1}, W_{2}\right)=0$ in $\mathbf{C}^{2} \times\left(\mathbf{C}^{*}\right)^{2}$ defined in (6.1). In Appendix A, we also construct explicitly the cycles which represent $\operatorname{mir}\left(\mathcal{O}_{p}\right)$ and $\operatorname{mir}\left(\mathcal{O}_{D}(-2)\right)$.

We remark that, in case of the two dimensional singularity, the hyperkähler rotation helped us identify the mirror cycles in $H_{2}(Y, \mathbf{Z})$. In the present case, however, it is not so obvious to see that there is a canonical (geometric) way to construct the isomorphism mir : $K^{c}(X) \stackrel{\sim}{\rightarrow} H_{3}(Y, \mathbf{Z})$. Nevertheless, our Conjecture 6.3 predicts that there is in fact a canonical isomorphism encoded indirectly in our cohomology-valued hypergeometric series. This situation is parallel to the mirror 
symmetry of compact Calabi-Yau manifolds, where Strominger-Yau-Zaslow construction provides a recipe for the canonical mirror correspondence.

Finally we determine the prepotential. For this, we construct the symplectic dual to $\mathcal{O}_{C}(-2)$ by $-\frac{1}{3} \mathcal{O}_{D}(-2)$. Then from (6.14) the special Kähler relation becomes as

$$
\left(1, t, \frac{\partial F}{\partial t}\right)=\left(1, w^{(1)}(x),-\frac{1}{3} w^{(2)}\right) .
$$

Integrating this relation, we obtain the Gromov-Witten invariants of the geometry $X=K_{\mathbf{P}^{2}}$ (, the first column of Table 1 below). It should be noted that the symplectic dual $\frac{1}{3} \mathcal{O}_{D}(-2)$ is no longer in $K^{c}(X)$ but in $K^{c}(X) \otimes \mathbf{Q}$.
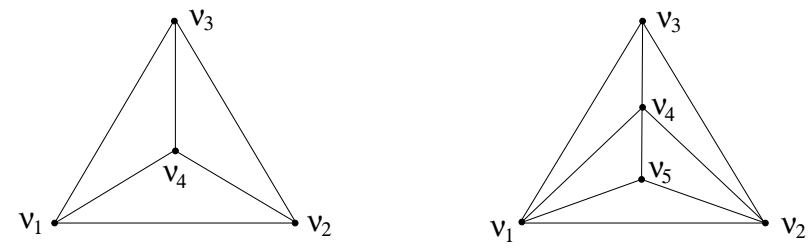

Fig.3. The resolution diagrams of the singularities $\mathbf{C}^{3} / G$ with $G=$ $\left\langle\frac{1}{3}(1,1,1)\right\rangle=\mathbf{Z}_{3}$ (left) and $G=\left\langle\frac{1}{5}(1,1,3)\right\rangle=\mathbf{Z}_{5}$ (right).

Example (2): As the second example, we briefly sketch the case $X=\widehat{\mathbf{C}^{3} / \mathbf{Z}_{5}}$ with the group action specified by the weights $\frac{1}{5}(1,1,3)$. The crepant resolution is described in Fig.3. The toric divisors $D_{4}:=D_{\nu_{4}}$ and $D_{5}:=D_{\nu_{5}}$ are identified, respectively, with the Hirzebruch surface $\mathbf{F}_{3}$ and $\mathbf{P}^{2}$. We will denote the rational curves that appear as the zero section of $\mathbf{F}_{3}$ by $b \equiv C_{1}$, and also the fiber by $f \equiv C_{2}$. Thus the intersection numbers on $\mathbf{F}_{3}$ are $b \cdot b=-3, b \cdot f=1, f \cdot f=0 . H^{2}(X, \mathbf{Z})$ is generated by $J_{1}$ and $J_{2}$ which measures the volumes $C_{1}=b$ and $C_{2}=f$, respectively (, i.e. $\int_{C_{i}} J_{k}=\delta_{i k}$ ). By the standard toric method (see, e.g., Oda2 [Fu]), we can determine the ring structure of the cohomology as

$$
H^{\text {even }}(X, \mathbf{Q})=\mathbf{Q}\left[J_{1}, J_{2}\right] /\left(J_{1}^{2}, J_{2}^{2}-3 J_{1} J_{2}, J_{2}^{3}\right) .
$$

The construction of the secondary fan $\operatorname{Sec}\left(\Sigma_{G}\right)$ is straightforward and we find $l^{(1)}=$ $(1,1,0,1,-3)$ and $l^{(2)}=(0,0,1,-2,1)$ for the local parameters $x_{1}=x:=a^{l^{(1)}}$ and $x_{2}=y:=a^{l^{(2)}}$ of the hypergeometric series. With these data we can write down the cohomology-valued hypergeometric series (6.4) with

$$
c(n, m)=\frac{1}{\Gamma(1+n)^{2} \Gamma(1+m) \Gamma(1+n-2 m) \Gamma(1-3 n+m)} .
$$

For the expansion of the cohomology-valued hypergeometric series, we first fix a symplectic D-brane basis of $K^{c}(X)$ by $\left\{\mathcal{B}_{J}\right\}=\left\{\mathcal{O}_{p}, \mathcal{O}_{b}\left(-J_{1}\right), \mathcal{O}_{f}\left(-J_{2}\right), \mathcal{O}_{\mathbf{P}^{2}}\left(-2 J_{1}\right)\right.$, $\left.\mathcal{O}_{\mathbf{F}_{3}}\left(-2 J_{2}\right)\right\}$. Then it is straightforward to express these bases in terms of $S_{k}$ 's by evaluating the pairings of $\mathcal{B}_{J}$ 's with the tautological bundles [Re] $\mathrm{Cr}$;

$$
\mathcal{F}_{0}=\mathcal{O}_{X}, \mathcal{F}_{1}=\mathcal{O}_{X}\left(J_{1}\right), \mathcal{F}_{2}=\mathcal{O}_{X}\left(2 J_{1}\right), \mathcal{F}_{3}=\mathcal{O}_{X}\left(J_{2}\right), \quad \mathcal{F}_{4}=\mathcal{O}_{X}\left(J_{1}+J_{2}\right) .
$$


S. HOSONO

Here we summarize the results together with their duals under the pairing $K^{c}(X) \times$ $K(X) \rightarrow \mathbf{Z}:$

$$
\begin{array}{cllr} 
& \multicolumn{1}{c}{K^{c}(X)} & \multicolumn{1}{c}{K(X)} \\
\mathcal{O}_{p} & =S_{0}+S_{1}+S_{2}+S_{3}+S_{4} & & -\mathcal{F}_{0}+2 \mathcal{F}_{1}+2 \mathcal{F}_{3}-2 \mathcal{F}_{4} \\
\mathcal{O}_{b}\left(-J_{1}\right) & =S_{1}+2 S_{2}+S_{4} & -\mathcal{F}_{3}+\mathcal{F}_{4} \\
\mathcal{O}_{f}\left(-J_{2}\right) & =S_{3}+S_{4} & \mathcal{F}_{0}-2 \mathcal{F}_{1}-\mathcal{F}_{3}+2 \mathcal{F}_{4} \\
\mathcal{O}_{\mathbf{P}^{2}}\left(-2 J_{1}\right) & =S_{2} & \mathcal{F}_{0}-2 \mathcal{F}_{1}+\mathcal{F}_{2} \\
\mathcal{O}_{\mathbf{F}_{3}}\left(-2 J_{2}\right) & =2 S_{0}+S_{1} & \mathcal{F}_{0}-\mathcal{F}_{1}-\mathcal{F}_{3}+\mathcal{F}_{4}
\end{array}
$$

The symplectic form on $K^{c}(X)$ may be evaluated using [Corollary 5.3. in IN] as

$$
\left(\chi\left(\mathcal{B}_{I}, \mathcal{B}_{J}\right)\right)=\left(\begin{array}{ccccc}
0 & 0 & 0 & 0 & 0 \\
0 & 0 & 0 & -3 & 1 \\
0 & 0 & 0 & 1 & -2 \\
0 & 3 & -1 & 0 & 0 \\
0 & -1 & 2 & 0 & 0
\end{array}\right)
$$

Evaluating the Chern characters of the tautological bundles in (6.15), we have for the basis $\left\{Q_{J}\right\}$ of $H^{e v e n}(X, \mathbf{Q})$,

$Q_{0}=1-2 J_{1} J_{2} ; Q_{2}^{1}=J_{1}+\frac{1}{2} J_{1}^{2}+J_{1} J_{2}, Q_{2}^{2}=J_{2}+\frac{7}{2} J_{1} J_{2} ; Q_{4}^{1}=J_{1}^{2}, Q_{4}^{2}=J_{1} J_{2}$,

by which we arrange the cohomology-valued hypergeometric series as

$$
w\left(x ; \frac{J}{2 \pi i}\right)=w^{(0)}(x) Q_{0}+\sum_{a=1}^{2} w_{a}^{(1)}(x) Q_{2}^{a}+\sum_{b=1}^{2} w_{b}^{(2)}(x) Q_{4}^{b},
$$

(where $w^{(0)}(x) \equiv 1$ from the form $c(n, m)$ above). Our Conjecture 6.3 predicts that the coefficient hypergeometric series $\left\{w^{(0)}(x), w_{a}^{(1)}(x), w_{b}^{(2)}(x)\right\}$ should be integral and symplectic with respect to the symplectic form (6.16).

As a check of the mirror symmetry, we determine the prepotential. First, we write the special Kähker relation (6.9),

$$
\left(1, t_{a}, \frac{\partial F}{\partial t_{a}}\right)=\left(1, w_{a}^{(1)}(x), \sum_{b} C^{a b} w_{b}^{(2)}(x)\right),
$$

with the matrix $\left(C^{a b}\right)=\left(\begin{array}{cc}-3 & 1 \\ 1 & -2\end{array}\right)^{-1}$. From this relation, it is straightforward to obtain

$$
\begin{aligned}
& \frac{\partial F}{\partial t_{1}}=-\frac{1}{5}\left(\partial_{\tilde{\rho}_{1}}^{2}+\partial_{\tilde{\rho}_{1}} \partial_{\tilde{\rho}_{2}}+\frac{3}{2} \partial_{\tilde{\rho}_{2}}^{2}\right) w(x)+\frac{2}{5} t_{1}+\frac{7}{10} t_{2}-\frac{2}{5}, \\
& \frac{\partial F}{\partial t_{2}}=-\frac{1}{10}\left(\partial_{\tilde{\rho}_{1}}^{2}+6 \partial_{\tilde{\rho}_{1}} \partial_{\tilde{\rho}_{2}}+9 \partial_{\tilde{\rho}_{2}}^{2}\right) w(x)+\frac{7}{10} t_{1}+\frac{27}{10} t_{2}-\frac{6}{5},
\end{aligned}
$$

where $\partial_{\tilde{\rho}_{k}}=\frac{1}{2 \pi i} \partial_{\rho_{k}}$. Just as the case for compact Calabi-Yau manifolds, we can integrate the special Kähler geometry relation with the prepotential

$F(t)=\frac{1}{6}\left(-\frac{2}{5} t_{1}^{3}-\frac{3}{5} t_{1}^{2} t_{2}-\frac{9}{5} t_{1} t_{2}^{2}-\frac{9}{5} t_{2}^{3}\right)+\frac{7}{10} t_{1} t_{2}+\frac{1}{5} t_{1}^{2}+\frac{27}{20} t_{2}^{2}-\frac{2}{5} t_{1}-\frac{6}{5} t_{2}+F_{\text {inst }}(t)$,

where $F_{\text {inst }}(t):=\sum_{n, m \geq 0} N(n, m) q_{1}^{n} q_{2}^{m}$ represents the quantum corrections. $N(n, m)$ is the genus zero Gromov-Witten invariants of the homology class $\beta=n b+m f \in$ $H_{2}(X, \mathbf{Z})$, i.e. $N(n, m)=N_{0}(n b+m f)$ in the standard notation $N_{g}(\beta)$ of GromovWitten invariants of genus $g$ in literatures. In Table 1 , we have listed the integral 
'invariants' called Gopakumar-Vafa invariants $\tilde{N}(n, m)$ GV]. One can verify that Gromov-Witten invariants coincides with the results from topological vertex technique (see AKMV]).

We may observe the similar integrability of the special Kähler relation for many other examples. In compact situations, this integrability (or more specifically the existence of the so-called Griffiths-Yukawa coupling) is a consequence of the Griffiths transversality (see $\underline{\mathrm{St}}$ ) for which we use an integration over (mirror) manifolds. However it should be noted that the existence of the prepotential for non-compact cases seems non-trivial, since integrating over a non-compact manifolds needs some special cares.

\begin{tabular}{|c|ccccccc|}
\hline $\mathrm{n} \backslash \mathrm{m}$ & 0 & 1 & 2 & 3 & 4 & 5 & 6 \\
\hline 0 & 0 & -2 & 0 & 0 & 0 & 0 & 0 \\
1 & 3 & 4 & 3 & 5 & 7 & 9 & 11 \\
2 & -6 & -10 & -12 & -12 & -24 & -56 & -140 \\
3 & 27 & 64 & 91 & 108 & 150 & 294 & 675 \\
4 & -192 & -572 & -980 & -1332 & -1808 & -2982 & -5992 \\
5 & 1695 & 6076 & 12259 & 18912 & 26983 & 42005 & 76608 \\
6 & -17064 & -71740 & -166720 & -289440 & -443394 & -689520 & -1192644 \\
7 & 188454 & 909760 & 2394779 & 4632120 & 7665776 & 12254816 & 20764870 \\
\hline
\end{tabular}

Table 1. Gopakumar-Vafa numbers $\tilde{N}_{0}(n, m)=\tilde{N}_{0}(n b+m f)$ for $X=\widehat{\mathbf{C}^{3} / \mathbf{Z}_{5}}$. $\tilde{N}_{0}(n, 0)$ coincides with the Gopakumar-Vafa numbers for $\widehat{\mathbf{C}^{3} / \mathbf{Z}_{3}}$.

\section{Conclusion and discussions}

We have studied in detail the cohomology-valued hypergeometric series for the case of non-compact Calabi-Yau manifolds. Giving an interpretation of $w\left(x ; \frac{J}{2 \pi i}\right)$ as the central charge formula (Conjecture 6.3), we have provided supporting evidences for the conjecture in the cases of $X=\mathbf{C}^{2 / \mathbf{Z}_{\mu+1}}$, and also $X=G$-Hilb $\mathbf{C}^{3}$ with finite abelian subgroups $G=\mathbf{Z}_{3}, \mathbf{Z}_{5} \subset S U(3, \mathbf{C})$. We have also clarified the structure of the prepotential for non-compact Calabi-Yau geometries $X=G$-Hilb $\mathbf{C}^{3}$.

As a byproduct of our study, we have found that the K.Saito's system of differential equations satisfied by the primitive forms may be replaced by a suitable (resonant and reducible) GKZ system, whose solutions we may set up easily.

As addressed after Conjecture 2.2 our cohomology-valued hypergeometric series (or the central charge formula) connects two different 'monodromy' properties, Fourier-Mukai transforms and the monodromy transforms of hypergeometric series (Dehn twists in the symplectic mapping class group). We see that the latter monodromy property arises associated with the discriminant locus in $\mathcal{M}_{\operatorname{Sec}(\Sigma)}$. 
As shown in section 3 for $X=\widehat{\mathbf{C}^{2} / \mathbf{Z}_{\mu+1}}$, the discriminant splits into several irreducible components in the $q$-coordinate and the monodromy transform around each irreducible component may be identified with a suitable twist functors. From these facts, it is conceivable that the group of self-equivalences of $D^{b}(\operatorname{Coh}(X))$, i.e. Auteq $D^{b}(X)$, is generated by these 'monodromy' transformations up to the shift functors,

$$
\text { Auteq } D^{b}\left(\mathbf{C}^{2 / \mathbf{Z}_{\mu+1}}\right) /\{[k] \mid k \in \mathbf{Z}\}=\left\langle R_{1}, \cdots, R_{\mu}, T_{\mathcal{O}_{C_{1}}(-1)}, \cdots, T_{\mathcal{O}_{C_{\mu}}(-1)}\right\rangle,
$$

where $R_{k}$ represents the functor tensoring the tautological line bundle $\mathcal{F}_{k}$ and $T_{\mathcal{O}_{C_{k}}(-1)}$ is the Seidel-Thomas twist. In Proposition 4.4 we have seen explicitly that these functors are represented by the corresponding actions on the roots $\beta_{k}(a)$ 's, i.e., changing the phases of the roots and permuting (braiding) of the roots $\beta_{k}(a)$ 's. This simple picture should provide us an intuition about the possible forms of the self-equivalences in $\operatorname{DFuk}(Y, \beta)$.

Similar correspondence between monodromy transforms and Fourier-Mukai transforms, and also the canonical mirror isomorphism mir : $K^{c}(X) \stackrel{\sim}{\rightarrow} H_{3}(Y, \mathbf{Z})$ are contained in our Conjecture 6.3 for three dimensional cases $X=\widehat{\mathbf{C}^{3} / G}=G$-Hilb $\mathbf{C}^{3}$ IN] BKR [CI]. In three dimensions, there can be several Calabi-Yau resolutions of the singularity $\mathbf{C}^{3} / G$, and correspondingly there are many different types of (Fourier-Mukai) transforms connecting these different resolutions [BKR] [CI]. It is interesting to study the details of the canonical mirror isomorphism mir from our cohomology-valued hypergeometric series in such situations.

As we have done for $X=\widehat{\mathbf{C}^{3} / \mathbf{Z}_{3}}$, in some cases with lower $\operatorname{dim} H^{2}(X, \mathbf{Q})$, we can determine explicitly the integral cycles and their monodromy properties to provid a consistency check for our Conjecture 6.3 We can also read the isomorphism mir : $K^{c}(X) \stackrel{\sim}{\rightarrow} H_{3}(Y, \mathbf{Z})$ from our cohomology-valued hypergeometric series. This isomorphism mir should also follow from the geometric framework like the Strominger-Yau-Zaslow construction, which provides a canonical equivalence Mir : $D_{c}^{b}(\operatorname{Coh}(X)) \stackrel{\sim}{\rightarrow} D F u k(Y, \beta)$.

Finally, as for the compact Calabi-Yau (hypersurfaces), several supporting evidences for our Conjecture 2.2 are reported in $\mathrm{Hos}$, especially for dimensions one and two. However, for example, the observation (1.4) made for quintic hypersurface $X_{5}$ is still needed a justification. Namely we have to work out a symplectic basis of the K-group $K\left(X_{5}\right)$ which brings the specific forms of the 'charges' $1, J-\frac{c_{1}\left(X_{5}\right) J}{12}-$ $\frac{11}{2} \frac{J^{2}}{5}, \frac{J^{2}}{5}, \frac{J^{3}}{5}$, and also their mirror cycles in $H_{3}\left(X_{5}^{\vee}, \mathbf{Z}\right)$ with their period integrals $w^{(0)}(x), w^{(1)}(x), w^{(2)}(x), w^{(3)}(x)$. From the SYZ construction [SYZ] LYZ] we may expect that $K\left(X_{5}\right)$ is generated by the structure sheaf $\mathcal{O}_{X}$, a skyscraper sheaf $\mathcal{O}_{p}$, and additional sheaves $\mathcal{E}$ and $\mathcal{F}$ which, respectively, have their support on a divisor and a curve (,i.e. $D 4$ and $D 2$ branes). The cycles which give the period integrals $w^{(0)}(x)$ and $w^{(3)}(x)$ are known in the original work CdOGP. In fact, claiming these cycles to be the mirror images of $\mathcal{O}_{p}$ and $\mathcal{O}_{X}$, i.e. the Lagrangian torus cycle $T^{3}$ and the section $S^{3}$ of the fibration, respectively, was the starting point of the SYZ construction. However we still lack our knowledge for the remaining cycles and also the corresponding sheaves $\mathcal{E}$ and $\mathcal{F}$. 


\section{APPENDIX A. Integrating over vanishing cycles}

(A-1) $\mathbf{C}^{2} / \mathbf{Z}_{\mu+1}$. As a warm up, let us first evaluate the period integral (4.2) of the primitive form, and then obtain the central charge $Z_{t}\left(S_{k}\right)$ in (5.4) of the sheaf $S_{k}=\mathcal{O}_{C_{i}}(-1)$ as an integral over the vanishing cycles $L_{i}=\operatorname{mir}\left(\mathcal{O}_{C_{i}}(-1)\right)$.

As in Proposition 4.2 we write the roots of the polynomial $\psi(W)=a_{0}+a_{1} W+$ $\cdots+a_{\mu+1} W^{\mu+1}$ by $\beta_{0}, \cdots, \beta_{\mu}$. Then for the defining equation of the singularity, we have

$$
f(a, W)+U^{2}+V^{2}=\left(W-\beta_{0}\right) \cdots\left(W-\beta_{\mu}\right)+U^{2}+V^{2},
$$

where we set $a_{\mu+1}=1$ by scaling the variable $W$. Assume that all the roots $\beta_{i}$ 's are real and $\beta_{0}<\beta_{1}<\cdots<\beta_{\mu}$. When $f(a, W) \leq 0$ holds for $\beta_{k-1} \leq W \leq \beta_{k}$, we may construct a vanishing cycle $L_{k}$ as $L_{k}=L_{k}^{+} \cup L_{k}^{-} \approx S^{2}$ with

$$
L_{k}^{ \pm}=\left\{\left( \pm \sqrt{|f(a, W)|-V^{2}}, V, W\right) \in \mathbf{R}^{3} \mid f_{\Sigma}(a, W)+V^{2} \leq 0, \beta_{k-1} \leq W \leq \beta_{k}\right\} .
$$

Similary, when $f(a, W) \geq 0$ for $\beta_{k-1} \leq W \leq \beta_{k}$, we construct vanishing cycles changing the variables; $U \rightarrow \sqrt{-1} U, V \rightarrow \sqrt{-1} V$. For the first case, the evaluation of the period integral over $L_{k}^{+}$proceeds as

$$
\begin{aligned}
& \int_{L_{k}^{+}} \operatorname{Res}_{F_{\Sigma}=0}\left(\frac{d W d U d V}{f(W)+U^{2}+V^{2}}\right)=\int_{\beta_{k-1}}^{\beta_{k}} d W \int_{-|f(W)|}^{|f(W)|} \frac{\left(\frac{1}{2}\right) d V}{\sqrt{|f(W)|-V^{2}}} \\
& =\left.\frac{1}{2} \int_{\beta_{k-1}}^{\beta_{k}} d W \operatorname{Sin}^{-1}\left(\frac{V}{\sqrt{|f(W)|}}\right)\right|_{-|f(W)|} ^{|f(W)|}=\frac{\pi}{2} \int_{\beta_{k-1}}^{\beta_{k}} d W,
\end{aligned}
$$

where $F_{\Sigma}:=f(W)+U^{2}+V^{2}$. We have a similar result for $L_{k}^{-}$, and in total $\pi\left(\beta_{k}-\beta_{k-1}\right)$ for the period integral. Since we have $\mu$-independent vanishing cycles in this way, we come to the well-known results summarized in Proposition 4.1 and also Proposition 4.2. In the above evaluation, one should note that the period integrals of the primitive form measures the volume of the vanishing cycles.

The period integral $\Pi_{\gamma}(a)$ (3.1) has slightly different shape in the measure of the integration. First let us take $\gamma=L_{k} \times S^{1}$ for the cycle $\gamma \in H_{3}\left(\mathbf{C}^{2} \times \mathbf{C}^{*} \backslash\left(F_{\Sigma}=0\right), \mathbf{Z}\right)$ with a loop $S^{1}$ which encircles the hypersurface. Since the integration over $S^{1}$ produces the holomorphic two form $\Omega\left(Y_{x}\right)$ used in (5.3), we have

$$
\int_{L_{k}^{+}} \Omega\left(Y_{x}\right)=\frac{1}{2} \int_{\beta_{k-1}}^{\beta_{k}} \frac{d W}{W} \int_{-|f(W)|}^{|f(W)|} \frac{d V}{\sqrt{|f(W)|-V^{2}}}=\frac{\pi}{2} \int_{\beta_{k-1}}^{\beta_{k}} \frac{d W}{W},
$$

and similar result for $L_{k}^{-}$. Thus, up to a normalization of $\Omega\left(Y_{x}\right)$, we arrive at the central charge of the sheaves $S_{k}=\mathcal{O}_{C_{k}}(-1)$ in (5.4) and justifies our claim that $\operatorname{mir}\left(\mathcal{O}_{C_{k}}(-1)\right)=L_{k}$ that follows from cohomology-valued hypergeometric series (5.3). One should note that the logarithmic behaviors of the solutions in Proposition 4.4 come from the torus invariant measure $\frac{d W}{W}$ in (3.1). The claimed relation $\operatorname{mir}\left(\mathcal{O}_{p}\right)=T_{0}$, with a cycle $T_{0} \approx S^{1} \times S^{1}$ will be justified below where we will define $T_{0}$ for three dimensional cases.

(A-2) $\mathbf{C}^{3} / \mathbf{Z}_{3}$. The evaluations of the period integrals in (A.1), A.2 extend to three dimensions. However, in three dimensions, the construction of the vanishing cycles becomes laborious as well as the integration over them. Moreover, the simple 
difference in the measure between the period integral A.1 of the primitive form and that of the local mirror symmetry A.2 makes the latter difficult to be integrated over cycles. Here, we will first consider the period integral over the primitive form, and then connect to that of local mirror symmetry defined in (6.2).

- Period integrals of the primitive form.

Let us start with the toric data for the crepant resolution $\widehat{\mathbf{C}^{3} / \mathbf{Z}^{3}}$, i.e., $\nu_{1}=$ $(1,0,0), \nu_{2}=(0,1,0), \nu_{3}=(0,0,1), \nu_{4}=(1 / 3,1 / 3,1 / 3)$ in $N_{G}$. We fix an isomorphism $\varphi: N_{G} \cong \mathbf{Z}^{3}$ such that $\varphi\left(\nu_{1}\right)=(1,0,0), \varphi\left(\nu_{2}\right)=(1,1,0), \varphi\left(\nu_{3}\right)=(1,2,3)$, $\varphi\left(\nu_{4}\right)=(1,1,1)$. Then consider the following deformation family of hypersurfaces in $\mathbf{C}^{4}$;

$$
F_{\Sigma}(a, U, V, W):=a_{1}+a_{2} W_{1}+a_{3} W_{1}^{2} W_{2}^{3}+a_{4} W_{1} W_{2}+U^{2}+V^{2}=0,
$$

with $\left(a_{1}, . ., a_{4}\right) \in\left(\mathbf{C}^{*}\right)^{4}$. The period integral of the primitive form is given by

$$
\int_{L} \mathcal{U}(a)=\int_{L} \operatorname{Res}_{F_{\Sigma}=0}\left(\frac{d U d V d W_{1} d W_{2}}{f_{\Sigma}(a, U, V, W)+U^{2}+V^{2}}\right),
$$

where $F_{\Sigma}(a, U, V, W)=f_{\Sigma}(a, W)+U^{2}+V^{2}$. As remarked after Proposition 6.2 this period integral satisfies the GKZ hypergeometric system (6.3) with the scaling operators $\mathcal{Z}_{2}, \mathcal{Z}_{3}$ replaced by $\mathcal{Z}_{2}^{\prime}=\mathcal{Z}_{2}+1$ and $\mathcal{Z}_{3}^{\prime}=\mathcal{Z}_{3}+1$, respectively. Then it is straightforward to see that the period integral

$$
\Pi_{L}^{\prime}(a):=\frac{1}{9 \pi} \frac{a_{4}^{2}}{a_{2} a_{3}} \int_{L} \mathcal{U}(a)
$$

is annihilated by the scaling operators $\mathcal{Z}_{i}(i=1,2,3)$, and thus a function of $x:=\frac{a_{1} a_{2} a_{3}}{a_{4}^{3}}$. (The numerical factor $\frac{1}{9 \pi}$ has been chosen just for later convenience.) As we have observed after Proposition 4.2 for two dimensions, the GKZ system of the primitive form is reducible and, after a factorization of a linear operator, we obtain the following differential equation for the irreducible part,

$$
\left\{\theta_{x}\left(\theta_{x}-1\right)+3 x\left(3 \theta_{x}-2\right)\left(3 \theta_{x}-1\right)\right\} \Pi_{L}^{\prime}(x)=0,
$$

where $\theta_{x}=x \frac{d}{d x}$ (, see Appendix B for the derivation). This reducible nature of the GKZ system for the primitive forms may be observed for other cases of the crepant resolutions $\widehat{\mathbf{C}^{3} / G}$ of an isolated singularity in $\mathbf{C}^{3} / G$.

Using the scaling property expressed by the equations $\mathcal{Z}_{i} \Pi_{L}^{\prime}(a)=0(i=1,2,3)$, we can reduce the parameters $\left(a_{1}, a_{2}, a_{3}, a_{4}\right) \in\left(\mathbf{C}^{*}\right)^{4}$ in $\Pi_{L}^{\prime}(a)$ to $(a, 1,1,-3)$. Namely one may reduce the defining equation $F_{\Sigma}(a, U, V, W)$ to the form

$$
a+W_{1}+W_{1}^{2} W_{2}^{3}-3 W_{1} W_{2}+U^{2}+V^{2}=: a+f_{1}(W)+U^{2}+V^{2} .
$$

Note that the parameter $a$ is related with the local parameter $x$ (in (6.10) by $x=-a / 27$.

Now, following general arguments on singularities AGV, we may construct vanishing cycles looking at the critical points of $f_{1}(W)$, (or more precisely $f_{1}(W)+$ $\left.U^{2}+V^{2}\right)$. We see that $f_{1}(W)$ has two critical points,

$$
\left(W_{1}, W_{2}\right)_{\text {crit }}=(1,1),(0,1 / 3),
$$

with their critical values -1 and 0 , respectively. The vanishing cycle near the critical point $(1,1)$ is easily constructed since the critical value of the polynomial 
$f_{1}(W)$ is a local minimum for real $\left(W_{1}, W_{2}\right)$ near $(1,1)$. Therefore for $0<a<1$, we have a vanishing cycle $L_{1}=L_{1}^{+} \cup L_{1}^{-} \approx S^{3}$,

$$
L_{1}^{ \pm}=\left\{\left(U, V, W_{1}, W_{2}\right) \in \mathbf{R}^{4} \mid \begin{array}{c}
U= \pm \sqrt{\left|a+f_{1}(W)\right|-V^{2}}, \quad a+f_{1}(W) \leq 0 \\
-\sqrt{\left|a+f_{1}(W)\right|} \leq V \leq \sqrt{\left|a+f_{1}(W)\right|}
\end{array}\right\} .
$$

The evaluation of the period integral may be done in a similar way to A.1 with the result;

$$
\Pi_{L_{1}}^{\prime}(a)=\frac{1}{\pi} \int_{L_{1}} \frac{d V d W_{1} d W_{2}}{2 \sqrt{\left|a+f_{1}(W)\right|-V^{2}}}=\int_{a+f_{1}(W) \leq 0} d W_{1} d W_{2} .
$$

Integrating this, and setting $t=1-a(=1+27 x)$, we obtain a series expansion;

$$
\Pi_{L_{1}}^{\prime}(a)=\frac{2 \pi}{\sqrt{3}}\left(t+\frac{t^{2}}{9}+\frac{10}{243} t^{3}+\frac{140}{6561} t^{4}+\cdots\right)=\frac{2 \pi}{\sqrt{3}} t_{2} F_{1}\left(\frac{1}{3}, \frac{2}{3}, 2, t\right) .
$$

Similarly, we should be able to evaluate the period integral for the cycle $L_{0}$ associated to the critical point $(0,1 / 3)$ with its critical value 0 . Here we will recourse this by making an analytic continuation of the period integral A.5 to $t=1$ (, i.e. $a=0$ ) and considering the monodromy transform of it. The necessary relation is the following simple analytic continuation of the hypergeometric series;

Proposition A.1. Consider a hypergeometric series $\phi(a):=a_{2} F_{1}\left(\frac{1}{3}, \frac{2}{3}, 2, a\right)$ for $|a|<1$. Then for the overlap $|a|<1$ and $|1-a|<1$, we have

$$
\frac{2 \pi}{\sqrt{3}} \phi(1-a)=\phi(a) \log \left(\frac{a}{27}\right)+\frac{9}{2}-a+\frac{2}{9} a^{2}+\frac{151}{1458} a^{3}+\cdots,
$$

where $\cdots$ represents the higher order terms whose general form is given below.

By the above relation (A.6), we see that the analytic continuation of $\Pi_{L_{1}}^{\prime}(a)=$ $\frac{2 \pi}{\sqrt{3}} \phi(1-a)$ in A.5 to $a=0$ is given by the right hand side of A.6), which we hereafter write by $h_{2}(x)(x=-a / 27)$. From the monodromy property of $h_{2}(x)$ around $x=0$, we obtain a hypergeometric series $h_{1}(x):=2 \pi i \phi(a)$. For the analytic continuation of $h_{1}(x)$ to $a=1$, we can use again the relation (A.6) for $2 \pi i \phi(a)=$ $2 \pi i \phi(1-(1-a))$. Thus we generate two independent solutions whose monodromy properties at $a=0$ and $a=1$ are determined by (A.6). Here, we present explicit expressions for the hypergeometric series $h_{1}(x), h_{2}(x)$ near $x=-a / 27=0$;

$$
\begin{aligned}
\frac{h_{1}(x)}{2 \pi i} & :=-27 \sum_{n=0}^{\infty} \frac{(-1)^{n-1} \Gamma(1+3(n-1))}{n \Gamma(n)^{3}} x^{n}=-27\left(x-3 x^{2}+30 x^{3}+\cdots\right) \\
h_{2}(x) & :=-\left.27 \frac{\partial}{\partial \rho} \sum_{n=0}^{\infty} \frac{(-1)^{n+\rho-1} \Gamma(1+3(n+\rho-1))}{\Gamma(1+n+\rho) \Gamma(n+\rho)^{2}} x^{n+\rho}\right|_{\rho=0} \\
& =\frac{h_{1}(x)}{2 \pi i} \log (-x)+\frac{9}{2}+27 x+162 x^{2}-\frac{4077}{2} x^{3}+\frac{62073}{2} x^{4}-\cdots .
\end{aligned}
$$

Using (A.6), it is easy to determine the monodromy matrices of $\left(h_{1}(x), h_{2}(x)\right)$ around $a=0,1, \infty$, respectively, to be

$$
M_{0}=\left(\begin{array}{ll}
1 & 0 \\
1 & 1
\end{array}\right), M_{1}=\left(\begin{array}{cc}
1 & -3 \\
0 & 1
\end{array}\right), M_{\infty}=\left(\begin{array}{cc}
1 & 3 \\
-1 & -2
\end{array}\right)
$$


S. HOSONO

where $M_{\infty}:=\left(M_{1} M_{0}\right)^{-1}$. As for the choice of $h_{1}(x)$ made above, we may find three other possibilities; $\left(-h_{1}(x), h_{2}(x)\right),\left( \pm 3 h_{1}(x), h_{2}(x)\right)$, for which we have integral monodromy matrices. Since the signs are simply related to the orientations of cycles, only the case $\left(3 h_{1}(x), h_{2}(x)\right)$ is non-trivial. However, it is conceivable to take the 'larger' integral structure, and thus to have $h_{1}(x)$ for the period integral $\Pi_{L_{0}}^{\prime}(a)$.

- Period integrals of the local mirror symmetry.

As we have seen in (A.1) and A.2, the difference of the period integral of the primitive form and that of local mirror symmetry is simply in the integration measure on $W_{1}, W_{2}$, depending on whether we consider these variables in $\mathbf{C}^{2}$ or $\left(\mathbf{C}^{*}\right)^{2}$. Although the difference is simple, the integration over the vanishing cycle $L_{1}$, for example, for the latter case is much complicated than (A.4). Also we have an additional cycle $T_{0}$ which gives trivial period integral 1 . This cycle is easily described when we change the variable as

$$
F_{\Sigma}=a+f_{1}(W)+U^{2}+V^{2}=a+f_{1}(W)+\tilde{U} \tilde{V} .
$$

This is the form used in HIV for the mirror geometry $Y$. The additional cycle is given by

$T_{0}:=\left\{\left(\tilde{U}, \tilde{V}, W_{1}, W_{2}\right) \in \mathbf{C}^{2} \times\left(\mathbf{C}^{*}\right)^{2}\left|\tilde{U}=\frac{-a-f_{1}(W)}{\tilde{V}},\right| \tilde{V}|=| W_{1}|=| W_{2} \mid=\varepsilon\right\}$.

Then, it is straightforward to evaluate the period integral,

$$
\int_{T_{0}} \operatorname{Res}_{F_{\Sigma}=0}\left(\frac{d \tilde{U} d \tilde{V}}{\tilde{U} \tilde{V}+a+f_{1}(W)} \frac{d W_{1} d W_{2}}{W_{1} W_{2}}\right)=\int_{|\tilde{V}|=\left|W_{1}\right|=\left|W_{2}\right|=\varepsilon} \frac{d \tilde{V}}{\tilde{V}} \frac{d W_{1} d W_{2}}{W_{1} W_{2}},
$$

which is a constant. Altogether, there are three period integrals for the local mirror case. They satisfy the well-studied (Picard-Fuchs) differential equation in literatures;

$$
\left\{\theta_{x}^{3}+3 x\left(3 \theta_{x}+2\right)\left(3 \theta_{x}+1\right) \theta_{x}\right\} \Pi(x)=0,
$$

which follows from the GKZ system in Proposition 6.2 One should note that the differential equation (A.8) is of order three while the corresponding differential equation A.3 is of order two. This difference is explained by the extra cycle $T_{0}$ constructed above. More precisely, we may formulate the following relations between the two differential equation.

Proposition A.2. 1) The space of the solutions of the third order equation (A.8) is mapped to that of the second order equation A.3) by the following linear transformation;

$$
\mathcal{T}[f(x)]:=\int_{0}^{x} \theta_{y} f(y) d y .
$$

2) The hypergeometric series $w^{(1)}(x)$ and $w^{(2)}(x)$ defined in (6.13) are mapped, up to a numerical factor, to $h_{1}(x)$ and $h_{2}(x)$ in A.7), respectively, by

$$
-27(2 \pi i)^{2} \mathcal{T}\left[w^{(k)}(x)\right]=h_{k}(x) \quad(k=1,2) .
$$


Proof. To derive the property 1$)$, we note a relations;

$\left(\theta_{x}-1\right)\left\{\theta_{x}\left(\theta_{x}-1\right)+3 x\left(3 \theta_{x}-2\right)\left(3 \theta_{x}-1\right)\right\} \mathcal{T}[f(x)]=x\left\{\theta_{x}^{2}+3 x\left(3 \theta_{x}+2\right)\left(3 \theta_{x}+1\right)\right\} \theta_{x} f(x)$, which follows from $\theta_{x} \mathcal{T}[f(x)]=x \theta_{x} f(x)$. Since the image $\operatorname{Im}(\mathcal{T})$ forms an (nontrivial) invariant subspace under the monodromy transformations, $\mathcal{T}[f(x)]$ should be annihilated by the second order differential operator. For the second property 2$)$, we simply evaluate the transforms for the power series $w^{(1)}(x), w^{(2)}(x)$ and compare with $h_{1}(x), h_{2}(x)$.

We identify the trivial solution, $w^{(0)}(x) \equiv 1$, with the period integral over the cycle $T_{0}$ with a suitable normalization of the holomorphic three form $\Omega\left(Y_{x}\right)$ of the mirror $Y$. Since this trivial solution is in the kernel of the map $\mathcal{T}$, we may identify our hypergeometric series $w^{(1)}(x), w^{(2)}(x)$ with the period integrals over the vanishing cycles over $L_{0}$ and $L_{1}$, respectively, up to adding some integral multiples of $\Pi_{T_{0}}(x)$. For the latter case, the property $w^{(2)}\left(-\frac{1}{27}\right)=0$, which one can verify easily, implies that $w^{(2)}(x)$ represents period integral over the vanishing cycle $L_{1}$. Similar argument does not apply to $w^{(1)}(x)$. However, the shift $w^{(1)}(x) \rightarrow w^{(1)}(x)+$ $n$ does not change the following monodromy matrices, we set this to zero and identify

$$
w^{(1)}(x)=\Pi_{L_{0}}(x), w^{(2)}(x)=\Pi_{L_{1}}(x),
$$

for the cycles $L_{0}, L_{1}$. It is straightforward to determine the monodromy matrices around $a=0,1, \infty$, respectively, of the solutions $\left(w^{(0)}(x), w^{(1)}(x), w^{(2)}(x)\right)$ with the results;

$$
M_{0}=\left(\begin{array}{lll}
1 & 0 & 0 \\
1 & 1 & 0 \\
0 & 1 & 1
\end{array}\right), M_{1}=\left(\begin{array}{ccc}
1 & 0 & 0 \\
0 & 1 & -3 \\
0 & 0 & 1
\end{array}\right), M_{\infty}=\left(\begin{array}{ccc}
1 & 0 & 0 \\
-1 & 1 & 3 \\
1 & -1 & -2
\end{array}\right)
$$

where $M_{\infty}:=\left(M_{1} M_{0}\right)^{-1}$. Now let us recall the relations (6.14) which follow from our Conjecture 6.3 From these, we may read the homological mirror map $\operatorname{mir}: K^{c}(X) \stackrel{\sim}{\rightarrow} H_{3}(Y, \mathbf{Z})$ as,

$$
\operatorname{mir}\left(\mathcal{O}_{p}\right)=T_{0}, \operatorname{mir}\left(\mathcal{O}_{C}(-1)\right)=L_{0}, \operatorname{mir}\left(\mathcal{O}_{D}(-2)\right)=L_{1} .
$$

We note that the sheaf $\mathcal{O}_{D}(-2)$ defines a spherical object (see [Proposition 3.19, $\mathrm{ST}]$ ). Under the homological mirror symmetry, spherical objects (, or some special class of the spherical objects, ) are expected to correspond to vanishing cycles. We have seen explicitly, in section 3, that this is the case for two dimensional cases. The above results A.11 in dimension three are consistent to this picture, and provides a support for our Conjecture 6.3 on the cohomology-valued hypergeometric series. 


\section{Appendix B. Differential equation (A.3)}

Here we derive the second order differential equation (A.3) form the GKZ system for the primitive form (cf. (6.3) ) observing a factorization of a differential operator. The same second order differential equation can be derived from the K.Saito's system.

As in (A-2), we fix the isomorphism $\varphi: N_{G} \widetilde{\sim} \mathbf{Z}^{3}$ so that

$$
\left(\varphi\left(\nu_{1}\right) \varphi\left(\nu_{2}\right) \varphi\left(\nu_{3}\right) \varphi\left(\nu_{4}\right)\right)=\left(\begin{array}{cccc}
1 & 1 & 1 & 1 \\
0 & 1 & 2 & 1 \\
0 & 0 & 3 & 1
\end{array}\right) .
$$

From this, we may write the linear operators $\mathcal{Z}_{i}^{\prime}$;

$$
\mathcal{Z}_{1}^{\prime}=\theta_{1}+\theta_{2}+\theta_{3}+\theta_{4}, \mathcal{Z}_{2}^{\prime}=\theta_{2}+2 \theta_{3}+\theta_{4}+1, \mathcal{Z}_{3}^{\prime}=3 \theta_{3}+\theta_{4}+1 .
$$

Since the period integral $\int_{C} \mathcal{U}(a)$ is annihilated by these operators, we have the following relations

$$
\theta_{2}=\theta_{1}-1, \theta_{3}=\theta_{1}-1, \theta_{4}=-3 \theta_{1}+2,
$$

when acting on $\int_{C} \mathcal{U}(a)$. Now consider the operator $\square_{l}$ for $l=(1,1,1,-3)$. We have

$$
a_{1} a_{2} a_{3} \square_{l} \int_{C} \mathcal{U}(a)=\left(\theta_{1} \theta_{2} \theta_{3}-\frac{a_{1} a_{2} a_{3}}{a_{4}^{3}}\left(\theta_{4}-2\right)\left(\theta_{4}-1\right) \theta_{4}\right) \int_{C} \mathcal{U}(a)=0 .
$$

Then it is easy to observe the following factorization of the operator when we eliminate $\theta_{a_{2}}, \theta_{a_{3}}, \theta_{a_{4}}$ by (B.1);

$$
\left(\theta_{1}-1\right)\left\{\theta_{1}\left(\theta_{1}-1\right)+3 \frac{a_{1} a_{2} a_{3}}{a_{4}^{3}}\left(3 \theta_{1}-2\right)\left(3 \theta_{1}-1\right)\right\} \int_{C} \mathcal{U}(a)=0 .
$$

The inhomogeneous terms (i.e. constants) in (B.1) may be removed if we define $\Pi_{C}^{\prime}(a):=\frac{a_{4}^{3}}{a_{2} a_{3}} \int_{C} \mathcal{U}(a)$. Even in that case, the shape of the above factorized differential operator does not change. The resulting homogeneous linear operators implies $\Pi_{C}^{\prime}(a)=\Pi_{C}^{\prime}(x)$ with $x=\frac{a_{1} a_{2} a_{3}}{a_{4}^{3}}$. Now we arrive at the second order differential equation,

$$
\left\{\theta_{x}\left(\theta_{x}-1\right)+3 x\left(3 \theta_{x}-2\right)\left(3 \theta_{x}-1\right)\right\} \Pi_{C}^{\prime}(x)=0,
$$

for the irreducible part. This completes our derivation of A.3.

Similar factorization property of the GKZ systems for primitive forms may be observed for $G$ such that $\mathbf{C}^{3} / G$ has an isolated singularity. This factorization property has also been observed in HKTY1 for the (extended) GKZ systems which appear in applications of mirror symmetry. 


\section{REFERENCES}

[AKMV] M. Aganagic, A. Klemm, M. Marino and Cumrun Vafa, The Topological Vertex , hep-th/0305132

[AGV] V.I. Arnold, S.M. Gusein-Zade and A.N. Varchenko, Singularities of Differential Maps, Vol.II, Birkhäuser (1988).

[Ba1] V.V. Batyrev, Dual polyhedra and mirror symmetry for Calabi-Yau hypersurfaces in toric varieties, J. Alg. Geom. 3 (1994) 493-535.

[Ba2] V.V. Batyrev, Variations of the mixed Hodge structure of affine hypersurfaces in algebraic tori, Duke Math. J. 69(1993), 349 - 409.

[Ba3] V.V. Batyrev, Talk given at Department of Mathematics, Tokyo Institute of Technology (September, 2003).

[Br] T. Bridgeland, Stability conditions on triangulated categories, math.AG/0212237

[BKR] T. Bridgeland, A. King and M. Reid, The McKay correspondence as an equivalence of derived categories, J. Amer. Math. Soc. 14(2001),no.3,535-554.

[CdOGP] P. Candelas, X.C. de la Ossa, P.S. Green, and L.Parkes, A pair of Calabi-Yau manifolds as an exactly soluble superconformal theory, Nucl.Phys. B356(1991), 21-74.

[CKYZ] T.-T. Chiang, A. Klemm, S.-T. Yau and E. Zaslow, Local Mirror Symmetry: Calculations and Interpretations, Adv.Theor.Math.Phys. 3(1999),495-565.

[Cr] A. Craw, An explicit construction of the McKay correspondence for A-Hilb $\mathbf{C}^{3}$, math.AG/0010053

[CI] A. Craw and A. Ishii, Flops of G-Hilb and equivalences of derived categories by variation of GIT quotient, math.AG/0211360

[dOFS] X. de la Ossa, B. Florea and H. Skarke, D-Branes on Noncompact Calabi-Yau Manifolds: K-Theory and Monodromy, Nucl.Phys. B644 (2002) 170-200.

[Do] M.R. Douglas, Dirichlet branes, homological mirror symmetry, and stability, math.AG/0207021 in the 2002 ICM proceedings.

[DFR] M. Douglas, B. Fiol and C. Römelsberger, The spectrum of BPS branes on a noncompact Calabi-Yau, hep-th/0003263

[FO3] K. Fukaya, Y.G. Oh, H. Ohta and K. Ono, Lagrangian intersection Floer theory - anomaly and obstruction -, preprint (2000) available at http://www.kusm.kyoto-u.ac.jp/ fukaya .

[Fu] W. Fulton, Introduction to Toric Varieties, Ann. of Math. Studies 131, Princeton University Press, Princeton, New Jersey, 1993.

[Gi] A.B. Givental, Equivariant Gromov-Witten invariants, Internat. Math. Res. Notices 13 (1996) 613-663.

[GKZ1] I.M. Gel'fand, A. V. Zelevinski, and M.M. Kapranov, Equations of hypergeometric type and toric varieties, Funktsional Anal. i. Prilozhen. 23(1989), 12 - 26; English transl. Functional Anal. Appl. 23(1989), 94-106.

[GKZ2] I.M. Gel'fand, A. V. Zelevinski, and M.M. Kapranov, Discriminants, Resultants and Multidimensional Determinants, Birkhäuser, Boston, 1994.

[GV] G. Gonzalez-Sprinberg and J.-L. Verdier, Construction géométrique de la correspondance de McKay, Ann. Sci. École Norm. Sup. 16(1983) 409-449.

[GoV] R. Gopakumar and C. Vafa, M-Theory and Topological Strings-II, hep-th/9812127

[GW] M. Gross and P.M.H. Wilson, Mirror symmetry via 3-tori for a class of Calabi-Yau threefolds, Math. Ann. 309 (1997) 505-531.

[HIV] K. Hori, A. Iqbal and C. Vafa, D-branes and mirror symmetry, hep-th/0005247

[Hor] P. Horja, Hypergeometric functions and mirror symmetry in toric varieties, math.AG/9912109

[Hos] S. Hosono, Local Mirror Symmetry and Type IIA Monodromy of Calabi-Yau Manifolds, Adv. Theor. Math. Phys. 4(2000), 335-376.

[HKTY1] S. Hosono, A. Klemm, S. Theisen, and S.-T. Yau, Mirror symmetry, mirror map and applications to Calabi-Yau hypersurfaces, Commun. Math. Phys. 167(1995), 301-350, hep-th/9308122

[HKTY2] S. Hosono, A. Klemm, S. Theisen, and S.-T. Yau, Mirror symmetry, mirror map and applications to complete intersection Calabi-Yau spaces, Nucl. Phys. B433 (1995) 501-554, hep-th/9406055 
[HLY] S. Hosono, B.H. Lian, and S.-T. Yau, GKZ-Generalized hypergeometric systems in mirror symmetry of Calabi-Yau hypersurfaces, Commun. Math. Phys. 182 (1996) 535577.

[Huy] D. Huybrechts, Moduli spaces of hyperkäher manifolds and mirror symmetry, math.AG/0210219

[IN] Y. Ito and H. Nakajima, The McKay correspondence and Hilbert schemes in dimension three, Topology 39 (2000), 1155-1191.

[Ko] M. Kontsevich, Homological algebra of mirror symmetry, Proceedings of the International Congress of Mathematicians (Zürich, 1994) Birkhäuser (1995) pp. 120 -139.

[LYZ] C.N. Leung, E. Zaslow and S.-T. Yau, From Special Lagrangian to Hermitian-YangMills via Fourier-Mukai Transform, Adv.Theor.Math.Phys. 4 (2002) 1319-1341.

[LLY] B.H. Lian, K. Liu and S.-T. Yau, Mirror principle I, Asian J. Math. 1 (1997), no. 4, 729-763, alg-geom/9712011

[Mor] D. R. Morrison, Picard-Fuchs equations and mirror maps for hypersurfaces, Essays on Mirror Manifolds (S.-T. Yau, ed.), Internal Press, Hong Kong, (1992), 241 - 264.

[Ma] P. Mayr, Phases of Supersymmetric D-branes on Kaehler Manifolds and the McKay correspondence,JHEP 0101 (2001) 018, hep-th/0010223

$[\mathrm{Mu}] \quad$ S. Mukai, On the moduli space of bundles on K3 surfaces I, in: Vector bundles on algebraic varieties Oxford Univ. Press (1987) 341-413.

[MR] S. Mukhopadhyay and K. Ray, Fractional branes on a noncompact orbifolds, JHEP 0107(2001)007.

[Na] I. Nakamura, Hilbert schemes of Abelian group orbits, J.Alg.Geom.10(2000)757-779.

[Oda1] T. Oda, Introduction to Algebraic Singularities (with an Appendix by T. Ambai), preprint (1989).

[Oda2] T. Oda, Convex bodies and Algebraic Geometry, An Introduction to the Theory of Toric Varieties, A Series of Modern Surveys in Mathematics, Springer-Verlarg New York, 1985.

[Or] D. Orlov, Equivalences of derived categories and K3 surfaces, math.AG/9606006 Algebraic geometry, 7. J. Math. Sci. (New York)84, no. 5 (1997) 1361-1381.

[Re] M. Reid, McKay correspondence, alg-geom/9702016

[Sa] K.Saito, Period Mapping Associated to a Primitive Form, Publ. RIMS, Kyoto Univ. 19(1983)1231-1264.

[ST] P. Seidel and R. Thomas, Braid group a actions on derived categories of coherent sheaves, math.AG/0001043 Duke Math. J. 108, no. 1 (2001) 37-108.

[Sti] J. Stienstra, Resonant hypergeometric systems and mirror symmetry, in Integrable systems and algebraic geometry (Kobe/Kyoto 1997), World Sci. Publishing, River Edge, NJ, 1998, 412-452.

[St] A. Strominger, Special Geometry, Commun. Math. Phys. 133 (1990) 163-180.

[SYZ] A. Strominger, S.-T. Yau and E. Zaslow Mirror symmetry is T-Duality, Nucl. Phys. B479 (1996) 243-259.

[Yo] M. Yoshida, Hypergeometric Functions, My Love, Vieweg (1997).

Graduate School of Mathematical Sciences, University of Tokyo, Komaba MeguroKU, TOKYO 153-8914, JAPAN

E-mail address: hosono@ms.u-tokyo.ac.jp 